\title{
Artificial Boundary Conditions for the Numerical Simulation of Unsteady Acoustic Waves
}

\author{
S. V. Tsynkov ${ }^{1}$ \\ Department of Mathematics and Center for Research in Scientific Computation \\ (CRSC), North Carolina State University, Box 8205, Raleigh, NC 27695, USA.
}

\begin{abstract}
We construct non-local artificial boundary conditions (ABCs) for the numerical simulation of genuinely time-dependent acoustic waves that propagate from a compact source in an unbounded unobstructed space. The key property used for obtaining the ABCs is the presence of lacunae, i.e., sharp aft fronts of the waves, in wave-type solutions in odd-dimension spaces. This property can be considered a manifestation of the Huygens' principle. The ABCs are obtained directly for the discrete formulation of the problem. They truncate the original unbounded domain and guarantee the complete transparency of the new outer boundary for all the outgoing waves. A central feature of the proposed $\mathrm{ABCs}$ is that the extent of their temporal nonlocality is fixed and limited, and it does not come at the expense of simplifying the original model. It is rather a natural consequence of the existence of lacunae, which is a fundamental property of the corresponding solutions. The proposed ABCs can be built for any consistent and stable finite-difference scheme. Their accuracy can always be made as high as that of the interior approximation, and it will not deteriorate even when integrating over long time intervals. Besides, the ABCs are most flexible from the standpoint of geometry and can handle irregular boundaries on regular grids with no fitting/adaptation needed and no accuracy loss induced. Finally, they allow for a wide range of model settings. In particular, not only one can analyze the simplest advective acoustics case with the uniform background flow, but also the case when the waves' source (or scatterer) is engaged in an accelerated motion.
\end{abstract}

Key words: Time-dependent sound waves, unbounded domains, lacunae, non-deteriorating method, long-term computation, limited temporal nonlocality.

Email address: tsynkov@math.ncsu.edu (S. V. Tsynkov).

URL: http://www. math.ncsu.edu/ stsynkov (S. V. Tsynkov).

1 The author gratefully acknowledges support by AFOSR, Grant F49620-01-1-0187, and that by NSF, Grant DMS-0107146. 


\section{Introduction}

Artificial Boundary Conditions (ABCs) is a common name for a group of methods employed for solving infinite-domain problems on a computer. ABCs facilitate truncation of the original unbounded domain, and provide the required closure for the resulting truncated formulation. The literature on the subject of $\mathrm{ABCs}$ is broad, and we refer the reader to the review papers [1-3].

Two opposite trends can be identified in building the ABCs. High accuracy requirements typically imply that the $\mathrm{ABCs}$ should be nonlocal. In particular, the exact $\mathrm{ABCs}$ are always nonlocal in multidimensional settings. For unsteady problems, this also means nonlocality in time. Clearly, nonlocality of the ABCs may result in high computational costs and elaborate implementation strategies. As such, local alternatives obtained either independently or as a approximations of nonlocal ABCs become viable. These ABCs are usually inexpensive and easy to implement, but may lack computational accuracy.

In the current paper we propose the ABCs for the numerical simulation of non-dispersive unsteady acoustic waves. We assume that there is a (complex) phenomenon/process confined to a bounded region that manifests itself by the radiation of acoustic waves in the far field. The waves subsequently propagate across the unbounded and unobstructed space, which is assumed isotropic and homogeneous. The proposed ABCs are nonlocal and guarantee that the accuracy of the boundary treatment can always be made at least as high as that of the interior discretization. However, the key feature of the proposed $\mathrm{ABCs}$ is that the extent of their temporal nonlocality is limited, and does not increase as the time elapses. The bound on temporal nonlocality comes as a consequence of the presence of lacunae, i.e., sharp aft fronts of the waves, in the solutions of the linearized Euler equations. In general, existence of the lacunae is a fundamental property of wave-type solutions in odd-dimension spaces. It is often referred to in connection with the Huygens' principle.

The ABCs are constructed by decomposing the original problem into the interior and auxiliary sub-problems. The latter is linear and homogeneous throughout the entire space, and is integrated with the help of lacunae. In so doing, the interior solution is used to generate sources for the auxiliary problem, and the auxiliary solution is used to provide the missing boundary data, i.e., the require closure, for the interior problem.

Several other nonlocal ABCs' methodologies for unsteady waves have been recently advocated in the literature, most notably [4-10]; we also mention the survey paper [11] and the bibliography there. Compared to these techniques, our approach has a number of distinctive characteristics. Its high accuracy and restricted temporal nonlocality have already been mentioned. Besides, the pro- 
posed lacunae-based ABCs are obtained directly for the discrete formulation of the problem, and can supplement any consistent and stable finite-difference scheme. In other words, they bypass the common stages of first deriving and then approximating the continuous ABCs, see [2]. The lacunae-based ABCs are especially designed to withstand long-term numerical integration with no deterioration of accuracy. Moreover, neither they are restricted to any particular shape of artificial boundary nor require grid fitting. They also enable analysis of a variety of problem formulations, e.g., the case of sound sources moving with acceleration, which is equivalent to advective acoustics with unsteady background flow. We note that the source motion does not have to be considered relative to an ambient medium only. Via the appropriate Galileo transform, it can be considered relative to a given mean flow as well.

The ABCs that we derive in the current paper for acoustics generalize and further extend our previous work $[12,13]$, in which a similar methodology was introduced for the scalar wave equation. The current approach also fits into the general theoretical framework of [14].

\section{Lacunae of the Wave Equation}

Consider a three-dimensional wave equation, $\boldsymbol{x}=\left(x_{1}, x_{2}, x_{3}\right)$ :

$$
\begin{gathered}
\frac{1}{c^{2}} \frac{\partial^{2} \varphi}{\partial t^{2}}-\left(\frac{\partial^{2} \varphi}{\partial x_{1}^{2}}+\frac{\partial^{2} \varphi}{\partial x_{2}^{2}}+\frac{\partial^{2} \varphi}{\partial x_{3}^{2}}\right)=f(\boldsymbol{x}, t), \quad t \geq 0, \\
\left.\varphi\right|_{t=0}=\left.\frac{\partial \varphi}{\partial t}\right|_{t=0}=0
\end{gathered}
$$

with homogeneous initial conditions. For every $(\boldsymbol{x}, t)$, the solution $\varphi=\varphi(\boldsymbol{x}, t)$ of problem (1a), (1b) is given by the Kirchhoff integral (see, e.g., [15]):

$$
\varphi(\boldsymbol{x}, t)=\frac{1}{4 \pi} \iiint_{\varrho \leq c t} \frac{f(\boldsymbol{\xi}, t-\varrho / c)}{\varrho} d \boldsymbol{\xi},
$$

where $\boldsymbol{\xi}=\left(\xi_{1}, \xi_{2}, \xi_{3}\right), \varrho=|\boldsymbol{x}-\boldsymbol{\xi}|=\sqrt{\left(x_{1}-\xi_{1}\right)^{2}+\left(x_{2}-\xi_{2}\right)^{2}+\left(x_{3}-\xi_{3}\right)^{2}}$, and $d \boldsymbol{\xi}=d \xi_{1} d \xi_{2} d \xi_{3}$. If we assume that the right-hand side (RHS) $f(\boldsymbol{x}, t)$ of equation (1a) is compactly supported in space-time on the domain $Q \subset$ $\mathbb{R}^{3} \times[0,+\infty)$, then formula (2) immediately implies that

$$
\varphi(\boldsymbol{x}, t) \equiv 0 \text { for }(\boldsymbol{x}, t) \in \bigcap_{(\boldsymbol{\xi}, \tau) \in Q}\{(\boldsymbol{x}, t)|| \boldsymbol{x}-\boldsymbol{\xi} \mid<c(t-\tau), t>\tau\} .
$$

The region of space-time defined by formula (3) is called lacuna of the solution $\varphi=\varphi(\boldsymbol{x}, t)$. This region is obtained as the intersection of all characteristic 
cones of equation (1a) once the vertex of the cone sweeps the support of the RHS: supp $f \subseteq Q$. From the standpoint of physics, lacuna corresponds to that part of space-time, on which the waves generated by the sources $f(\boldsymbol{x}, t)$, supp $f \subseteq Q$, have already passed, and the solution has become zero again. The phenomenon of lacunae is inherently three-dimensional. The surface of the lacuna represents the trajectory of aft (trailing) fronts of the waves. The existence of aft fronts in odd-dimension spaces is known as the Huygens' principle, as opposed to the so-called wave diffusion which takes place in evendimension spaces, see, e.g., [15]. Let us also note that the aft fronts and the lacunae would still be present in the solution of the wave equation (1a) if the homogeneous initial conditions (1b) were replaced by some inhomogeneous initial conditions with compact support.

The notion of lacunae (or lacunas) was first introduced and studied by Petrowsky in [16] (see also an account in [17, Chapter VI]) for a variety of hyperbolic equations and systems; and general characterization of their coefficients was provided that would guarantee existence of the lacunae. However, since work [16] no constructive examples of either equations or systems have been obtained for which lacunae would be present in the solutions, besides the actual wave equation (1a), as well as those equations that either reduce to or are derived from, the wave equation.

\section{The Acoustics System of Equations}

The acoustics (linearized Euler's) system in its simplest form governs the propagation of sound in an ambient compressible fluid [18, Chapter VIII]:

$$
\begin{aligned}
\frac{1}{c^{2}} \frac{\partial p}{\partial t}+\rho_{0} \nabla \cdot \boldsymbol{u} & =\rho_{0} q_{\mathrm{vol}} \\
\rho_{0} \frac{\partial \boldsymbol{u}}{\partial t}+\nabla p & =\boldsymbol{b}_{\mathrm{vol}} .
\end{aligned}
$$

In (4), $c$ is the speed of sound, and the variables $\boldsymbol{u}=\boldsymbol{u}(\boldsymbol{x}, t)$ and $p=p(\boldsymbol{x}, t)$ represent small perturbations of the velocity and pressure, respectively. Adiabatic law $p=c^{2} \rho$ has been applied that relates $p$ to the density perturbation $\rho$. The quantity $\rho_{0}$ is the constant background density. The source term $q_{\mathrm{vol}}=q_{\mathrm{vol}}(\boldsymbol{x}, t)$ that alters the balance of mass in the system is known as volume velocity per unit volume, and the source term $\boldsymbol{b}_{\mathrm{vol}}=\boldsymbol{b}_{\mathrm{vol}}(\boldsymbol{x}, t)$ that alters the balance of momentum is known as force per unit volume (see, e.g., [19]).

Proposition 1 Assume that the initial velocity field is conservative: $\boldsymbol{u}(\boldsymbol{x}, 0)=\nabla \varphi_{0}(\boldsymbol{x})$. Then, the velocity potential exists in the solutions of system (4) for all $t \geq 0$ if and only if the forcing $\boldsymbol{b}_{\mathrm{vol}}$ is also conservative: $\boldsymbol{b}_{\mathrm{vol}}(\boldsymbol{x}, t)=\nabla \psi(\boldsymbol{x}, t)$. In this case, the potential satisfies the wave equation. 
PROOF. Let us assume that the force field is conservative, and integrate the second equation of system (4) with respect to time starting from $t=0$ :

$$
\begin{aligned}
\rho_{0} \boldsymbol{u}(\boldsymbol{x}, t) & =\rho_{0} \boldsymbol{u}(\boldsymbol{x}, 0)-\int_{0}^{t} \nabla p(\boldsymbol{x}, \tau) d \tau+\int_{0}^{t} \boldsymbol{b}_{\mathrm{vol}}(\boldsymbol{x}, \tau) d \tau \\
& =\rho_{0} \nabla \varphi_{0}(\boldsymbol{x})-\int_{0}^{t} \nabla p(\boldsymbol{x}, \tau) d \tau+\int_{0}^{t} \nabla \psi(\boldsymbol{x}, \tau) d \tau \\
& =\nabla\left[\rho_{0} \varphi_{0}(\boldsymbol{x})-\int_{0}^{t} p(\boldsymbol{x}, \tau) d \tau+\int_{0}^{t} \psi(\boldsymbol{x}, \tau) d \tau\right] \\
& \stackrel{\text { def }}{=} \rho_{0} \nabla \varphi(\boldsymbol{x}, t) .
\end{aligned}
$$

Therefore, we obtain:

$$
\boldsymbol{u}(\boldsymbol{x}, t)=\nabla \varphi(\boldsymbol{x}, t), \quad \rho_{0} \frac{\partial \varphi(\boldsymbol{x}, t)}{\partial t}=-p(\boldsymbol{x}, t)+\psi(\boldsymbol{x}, t)
$$

and by differentiating the second relation in (6) w.r.t. time and subsequently substituting it into the first equation of system (4), we conclude that the potential $\varphi=\varphi(\boldsymbol{x}, t)$ defined by (5) will satisfy the wave equation (1a) with the RHS given by $f(\boldsymbol{x}, t)=-q_{\mathrm{vol}}(\boldsymbol{x}, t)+\frac{1}{\rho_{0} c^{2}} \frac{\partial \psi(\boldsymbol{x}, t)}{\partial t}$.

Conversely, let us assume that the velocity field is conservative: $\boldsymbol{u}(\boldsymbol{x}, t)=$ $\nabla \varphi(\boldsymbol{x}, t)$. Then, the momentum equation in system (4) can be recast as follows:

$$
\nabla\left[\rho_{0} \frac{\partial \varphi}{\partial t}+p\right]=\boldsymbol{b}_{\mathrm{vol}}
$$

which means that the forcing must be conservative: $\boldsymbol{b}_{\mathrm{vol}}(\boldsymbol{x}, t)=\nabla \psi(\boldsymbol{x}, t)$.

Proposition 1 also implies that if $\boldsymbol{b}_{\mathrm{vol}}(\boldsymbol{x}, t) \equiv 0$ then the potential always exists. In this case, the second equality of (6) reduces to the conventional relation between the potential and pressure: $\rho_{0} \frac{\partial \varphi}{\partial t}=-p$, see [18, Chapter VIII].

Applying the gradient operator to the wave equation for the potential, we conclude that the velocity vector also satisfies the wave equation:

$$
\frac{1}{c^{2}} \frac{\partial^{2} \boldsymbol{u}}{\partial t^{2}}-\Delta \boldsymbol{u}=-\nabla q_{\mathrm{vol}}+\frac{1}{\rho_{0} c^{2}} \frac{\partial \boldsymbol{b}_{\mathrm{vol}}}{\partial t} .
$$

Next, by differentiating the first equation of (4) with respect to time, taking the divergence of the second equation of (4), and substituting the result into the first one, we arrive at the wave equation for $p$ :

$$
\frac{1}{c^{2}} \frac{\partial^{2} p}{\partial t^{2}}-\Delta p=-\nabla \cdot \boldsymbol{b}_{\mathrm{vol}}+\rho_{0} \frac{\partial q_{\mathrm{vol}}}{\partial t}
$$


The key consideration of interest is that if the RHSs of system (4) are compactly supported in space and time on some domain $Q \subset \mathbb{R}^{3} \times[0,+\infty)$, then the RHSs of both equation (7) and equation (8) will also be compactly supported on the same domain $Q$. Consequently, solutions of equations (7) and (8) will have lacunae of the same shape as prescribed by formula (3). Thus, we have arrived at the following

Proposition 2 Let the RHSs of system (4) be compactly supported in space and time: $\operatorname{supp} q_{\mathrm{vol}}(\boldsymbol{x}, t) \subseteq Q$ and $\operatorname{supp} \boldsymbol{b}_{\mathrm{vol}}(\boldsymbol{x}, t) \subseteq Q$, where $Q \subset \mathbb{R}^{3} \times$ $[0,+\infty)$. Let also $\boldsymbol{u}(\boldsymbol{x}, t)=\nabla \varphi(\boldsymbol{x}, t)$. Then, solutions of the acoustics system (4) with homogeneous initial conditions will have lacunae of the same geometry as provided by formula (3).

In other words, we have shown that existence of the velocity potential is sufficient for the acoustics system (4) to have lacunae in its solutions; and that the geometry of the lacunae is determined by that of the support of the RHSs, as in the case of the wave equation. We do not know whether this condition is also necessary for having the lacunae. However, in the view of the comment provided in the end of Section 2, we will hereafter be using the foregoing sufficient condition as the only reliable indication of the presence of lacunae in the solutions of system (4).

The group of numerical algorithms for acoustics that we describe hereafter will be essentially based on the presence of lacunae. However, the Kirchhoff formula (2) will never be used as an actual part of the algorithm construction, it will only be needed at the theoretical stage, for determining the shape of the lacunae that will later be incorporated into a purely finite-difference context. Previously, the idea of using the Huygens' principle for constructing the ABCs was promoted by Ting \& Miksis [20] and Givoli \& Cohen [21]. Both papers, however, have suggested to use numerical quadratures to approximate the integral (2), and then couple it with the interior solution. Moreover, the approach of [20] has never been actually implemented in a practical computational setting, whereas the approach of [21] required artificial dissipation to be added to the scheme to fix the arising instabilities.

\section{Lacunae-Based Integration of the Acoustics System}

We will be looking for an irrotational solution of system (4) on a bounded domain $S=S(t) \subset \mathbb{R}^{3}$. We assume that this is a domain of fixed shape that has a finite diameter $d$ for all $t \geq 0$. We also assume that $\forall t \geq 0$ : $\left\{\operatorname{supp} q_{\text {vol }}(\boldsymbol{x}, t) \cap \mathbb{R}^{3}\right\} \subseteq S(t) \&\left\{\operatorname{supp} \boldsymbol{b}_{\text {vol }}(\boldsymbol{x}, t) \cap \mathbb{R}^{3}\right\} \subseteq S(t)$. In other words, we want to compute the acoustic field on a given domain that also contains all the field sources. While always remaining finite in size, this domain $S(t)$ is 
allowed to move across the space $\mathbb{R}^{3}$ according to a general law:

$$
\boldsymbol{u}_{0}=\boldsymbol{u}_{0}(t), \quad \boldsymbol{x}_{0}=\boldsymbol{x}_{0}(t)=\int_{0}^{t} \boldsymbol{u}_{0}(\tau) d \tau
$$

where $\boldsymbol{u}_{0}$ and $\boldsymbol{x}_{0}$ are the velocity vector and coordinates of a given point inside $S(t)$, respectively. The only limitation that we impose is that motion (9) be subsonic: $\max _{t}\left|\boldsymbol{u}_{0}(t)\right|=k<c$. Let now $\tilde{q}_{\mathrm{vol}}$ and $\tilde{\boldsymbol{b}}_{\mathrm{vol}}$ be some stationary acoustic sources (RHSs to system (4)), i.e., compactly supported functions of $\boldsymbol{x}$ on the fixed domain $S(0)$ for all $t \geq 0$ : $\left\{\operatorname{supp} \tilde{q}_{\mathrm{vol}}(\boldsymbol{x}, t) \cap \mathbb{R}^{3}\right\} \subseteq$ $S(0) \&\left\{\operatorname{supp} \tilde{\boldsymbol{b}}_{\mathrm{vol}}(\boldsymbol{x}, t) \cap \mathbb{R}^{3}\right\} \subseteq S(0)$. Then, we can incorporate the translational motion (9) into the acoustics system (4) as follows, see [12]:

$$
\begin{aligned}
\frac{1}{c^{2}} \frac{\partial p(\boldsymbol{x}, t)}{\partial t}+\rho_{0} \nabla \cdot \boldsymbol{u}(\boldsymbol{x}, t) & =\rho_{0} \tilde{q}_{\mathrm{vol}}\left(\boldsymbol{x}-\boldsymbol{x}_{0}(t), t\right) \equiv \rho_{0} q_{\mathrm{vol}}(\boldsymbol{x}, t), \\
\rho_{0} \frac{\partial \boldsymbol{u}(\boldsymbol{x}, t)}{\partial t}+\nabla p(\boldsymbol{x}, t) & =\tilde{\boldsymbol{b}}_{\mathrm{vol}}\left(\boldsymbol{x}-\boldsymbol{x}_{0}(t), t\right) \equiv \boldsymbol{b}_{\mathrm{vol}}(\boldsymbol{x}, t) .
\end{aligned}
$$

With no loss of generality, the initial conditions for integrating system (10) will always be assumed homogeneous. It is easy to see from (10) that the timedependent nature of the acoustic field is caused by the motion of the sound sources, on which the genuine unsteadiness of the sound generating mechanisms may be "superimposed." The domain of integration $S(t)$ has a fixed size/shape and traces the motion of the sources. As shown in [12, Appendix] using the Galileo transform, system (10) is equivalent to advective acoustics with stationary sources and unsteady background flow $-\boldsymbol{u}_{0}=-\boldsymbol{u}_{0}(t)$.

Let us emphasize that the setup we have introduced is quite general and also includes the case of the waves' sources that move relative to a given mean flow (rather than relative to the ambient fluid only). Indeed, a similar argument based on the Galileo transform would imply that having the mean flow $\boldsymbol{v}_{0}$ and the source motion $\boldsymbol{u}_{0}$ on top of it is equivalent to either stationary sources in the mean flow $\boldsymbol{v}_{0}-\boldsymbol{u}_{0}$ or alternatively, sources that move with the velocity $-\boldsymbol{v}_{0}+\boldsymbol{u}_{0}$ through the ambient medium. The latter setup is obviously the same as (9), as long as the condition $\max _{t}\left|-\boldsymbol{v}_{0}(t)+\boldsymbol{u}_{0}(t)\right|=k<c$ is met.

The case of primary interest for our analysis will be that of continuously operating sources in (10), $t \in[0,+\infty)$. Let us, however, assume for the time being that not only the RHSs in system (10) are compactly supported in space, but also that their "lifespan" in time is finite: $\operatorname{supp} q_{\mathrm{vol}}(\boldsymbol{x}, t) \in Q$ and supp $\boldsymbol{b}_{\mathrm{vol}}(\boldsymbol{x}, t) \in Q$, where $\mathbb{R}^{3} \times[0,+\infty) \ni Q=\left\{(\boldsymbol{x}, t) \mid \boldsymbol{x} \in S(t), t_{0} \leq t \leq t_{1}\right\}$. Then, Proposition 2 implies that no later than

$$
t=t_{2} \equiv t_{0}+\frac{d+\left(t_{1}-t_{0}\right)(c+k)}{c-k} \equiv t_{0}+T_{\mathrm{int}}
$$

all of the domain $S(t)$ will fall into the lacuna defined by formula (3) (see 
$[12,13]$ for a more detailed argument), and will remain inside the lacuna continuously thereafter, i.e., for all $t \geq t_{2}$. In other words, for the solution of system (10), we will have $p(\boldsymbol{x}, t)=0$ and $\boldsymbol{u}(\boldsymbol{x}, t)=\boldsymbol{O}$ when $\boldsymbol{x} \in S(t)$ and $t \geq t_{2}$, where $t_{2}$ is defined by formula (11).

Next, we realize that during the time interval $T_{\text {int }}$ no wave can travel in space further away than the distance $c T_{\text {int }}$ from the boundary of the domain $S\left(t_{0}\right)$. This means that we will also have $p(\boldsymbol{x}, t)=0$ and $\boldsymbol{u}(\boldsymbol{x}, t)=\boldsymbol{O}$ for $\operatorname{dist}\left(\boldsymbol{x}, S\left(t_{0}\right)\right)>c T_{\text {int }}$ and $t_{0} \leq t \leq t_{2}$. As such, instead of the free unobstructed space outside $S(t)$ we may consider outer boundaries with arbitrary (reflecting) properties. As long as none of these boundaries is located closer than $c T_{\text {int }}$ to $S\left(t_{0}\right)$, the solution of (10) inside $S(t)$ is not going to feel their presence for $t_{0} \leq t \leq t_{2}$.

In fact, the foregoing limitation for the location of outer boundaries can even be relaxed if instead of requiring that no wave may reach an outer boundary before $t=t_{2}$ we introduce a weaker requirement that no reflected wave may reach $S(t)$ before $t=t_{2}$. The latter consideration easily translates into the following estimate for the minimal distance between the domain $S\left(t_{0}\right)$ and the allowed location of any reflecting boundary (see $[12,13]$ for more detail):

$$
Z_{\min }=\frac{c+k}{2} T_{\mathrm{int}}
$$

We note that both estimates (11) and (12) are conservative in the sense that they do not take into account the direction of the motion. In case the motion of the sources is characterized by a specific or predominant direction, then the quantity $Z_{\min }$ can be further reduced in the orthogonal directions.

Altogether we conclude that the solution of system (10) driven by the sources compactly supported on the domain $Q=\left\{(\boldsymbol{x}, t) \mid \boldsymbol{x} \in S(t), t_{0} \leq t \leq t_{1}\right\}$ and subject to the homogeneous initial conditions, can be obtained on $S(t)$ for all $t \geq 0$ as follows. First, this solution is obviously equal to zero for $0 \leq t<t_{0}$. Next, on the time interval $t_{0} \leq t \leq t_{2}$, see formula (11), system (10) should be integrated on the auxiliary domain of size

$$
Z=d+2 Z_{\min }=d+(c+k) T_{\mathrm{int}}
$$

centered around $S\left(t_{0}\right)$, which in any event is going to yield the correct solution inside $S(t)$. Finally, for all $t \geq t_{2}$ the solution on $S(t)$ will be equal to zero again because all the waves will have left the domain by $t=t_{2}$ (lacuna).

Let us now address the case of continuously operating sources. For that pur- 
pose, we introduce a partition of unity on the semi-infinite interval $t \geq 0$ :

$$
\forall t \geq 0: \quad \sum_{j=0}^{\infty} \Theta(t-\sigma T j)=1
$$

where $T>0$ and $\frac{1}{2} \leq \sigma<1$ are two parameters, and $\Theta=\Theta(t)$ is a smooth, even, "hat"-type function with $\operatorname{supp} \Theta(t) \subseteq\left[-\frac{T}{2}, \frac{T}{2}\right]$ :

$$
\Theta(t)= \begin{cases}0, & t>\frac{T}{2} \\ 1, & 0 \leq t \leq\left(\sigma-\frac{1}{2}\right) T \\ 1-\Theta(\sigma T-t), & \left(\sigma-\frac{1}{2}\right) T<t \leq \frac{T}{2} \\ \Theta(-t), & t<0\end{cases}
$$

Then, we define compactly supported sources for $j=0,1,2, \ldots$ :

$$
\begin{array}{cc}
q_{\mathrm{vol}}^{(j)}(\boldsymbol{x}, t)=q_{\mathrm{vol}}(\boldsymbol{x}, t) \Theta(t-\sigma T j), & \operatorname{supp} q_{\mathrm{vol}}^{(j)}(\boldsymbol{x}, t) \subseteq Q_{j}, \\
\boldsymbol{b}_{\mathrm{vol}}^{(j)}(\boldsymbol{x}, t)=\boldsymbol{b}_{\mathrm{vol}}(\boldsymbol{x}, t) \Theta(t-\sigma T j), & \operatorname{supp} \boldsymbol{b}_{\mathrm{vol}}^{(j)}(\boldsymbol{x}, t) \subseteq Q_{j},
\end{array}
$$

where according to (15):

$$
Q_{j}=\left\{(\boldsymbol{x}, t) \mid \boldsymbol{x} \in S(t),\left(\sigma j-\frac{1}{2}\right) T \leq t \leq\left(\sigma j+\frac{1}{2}\right) T\right\}
$$

and consider a collection of sub-problems driven by the RHSs (16):

$$
\begin{aligned}
\frac{1}{c^{2}} \frac{\partial p^{(j)}}{\partial t}+\rho_{0} \nabla \cdot \boldsymbol{u}^{(j)} & =\rho_{0} q_{\mathrm{vol}}^{(j)}, & \rho_{0} \frac{\partial \boldsymbol{u}^{(j)}}{\partial t}+\nabla p^{(j)} & =\boldsymbol{b}_{\mathrm{vol}}^{(j)}, \\
\left.p^{(j)}(\boldsymbol{x}, t)\right|_{t=\left(\sigma j-\frac{1}{2}\right) T} & =0, & \left.\boldsymbol{u}^{(j)}(\boldsymbol{x}, t)\right|_{t=\left(\sigma j-\frac{1}{2}\right) T} & =0 .
\end{aligned}
$$

As formula (14) is a partition of unity, we have:

$$
q_{\mathrm{vol}}(\boldsymbol{x}, t)=\sum_{j=0}^{\infty} q_{\mathrm{vol}}^{(j)}(\boldsymbol{x}, t) \quad \text { and } \quad \boldsymbol{b}_{\mathrm{vol}}(\boldsymbol{x}, t)=\sum_{j=0}^{\infty} \boldsymbol{b}_{\mathrm{vol}}^{(j)}(\boldsymbol{x}, t),
$$

and because of the linear superposition, the solution $p(\boldsymbol{x}, t), \boldsymbol{u}(\boldsymbol{x}, t)$ of system (10) subject to the homogeneous initial conditions at $t=0$ can be expanded in terms of the solutions to systems (18):

$$
p(\boldsymbol{x}, t)=\sum_{j=0}^{\infty} p^{(j)}(\boldsymbol{x}, t) \quad \text { and } \quad \boldsymbol{u}(\boldsymbol{x}, t)=\sum_{j=0}^{\infty} \boldsymbol{u}^{(j)}(\boldsymbol{x}, t) .
$$

The series (19) are formally infinite. However, for any $t>0$ and $\boldsymbol{x} \in S(t)$ each will, in fact, contain only a finite fixed number of nonzero terms. Indeed, due to the causality for a given $t>0$ and all $\left(\sigma j-\frac{1}{2}\right) T>t$, i.e., all $j>\left(\frac{t}{T}+\frac{1}{2}\right) / \sigma$, we will have $p^{(j)}(\boldsymbol{x}, t)=0$ and $\boldsymbol{u}^{(j)}(\boldsymbol{x}, t)=\boldsymbol{O}$ on the entire space $\mathbb{R}^{3}$. Moreover, 
multiplication by the function $\Theta$, see (16), that is only a function of time will not alter the conservative nature of $\boldsymbol{b}_{\mathrm{vol}}$. Therefore, Proposition 2 will apply to the solution of every problem $(18), j=0,1,2, \ldots$ As such, if we interpret the moment $\left(\sigma j-\frac{1}{2}\right) T$ of the inception of source $j$ as $t_{0}^{(j)}$, and the moment $\left(\sigma j+\frac{1}{2}\right) T$ of its cessation as $t_{1}^{(j)}$, then according to formula (11):

$$
\begin{gathered}
\forall t>\left(\sigma j-\frac{1}{2}\right) T+\frac{d+T(c+k)}{c-k} \equiv t_{0}^{(j)}+T_{\mathrm{int}}: \\
p^{(j)}(\boldsymbol{x}, t)=0 \text { and } \boldsymbol{u}^{(j)}(\boldsymbol{x}, t)=0 \text { for } \boldsymbol{x} \in S(t),
\end{gathered}
$$

i.e., the domain $S(t)$ will be entirely inside the lacuna starting from $t_{2}^{(j)}=$ $t_{0}^{(j)}+T_{\text {int }}$. Alternatively, this means that for any $t>0$ and all $j<\left(\frac{t-T_{\text {int }}}{T}+\frac{1}{2}\right) / \sigma$, where $T_{\text {int }}=\frac{d+T(c+k)}{c-k}$ as in formula $(20)$, the terms $p^{(j)}(\boldsymbol{x}, t)$ and $\boldsymbol{u}^{(j)}(\boldsymbol{x}, t)$ in the series (19) will be equal to zero for $\boldsymbol{x} \in S(t)$. Consequently, for all $t>0$ and $\boldsymbol{x} \in S(t)$ we can replace expansions (19) with

$$
p(\boldsymbol{x}, t)=\sum_{j=m_{1}}^{m_{2}} p^{(j)}(\boldsymbol{x}, t) \quad \text { and } \quad \boldsymbol{u}(\boldsymbol{x}, t)=\sum_{j=m_{1}}^{m_{2}} \boldsymbol{u}^{(j)}(\boldsymbol{x}, t),
$$

where $m_{1}=\left[\left(\frac{t-T_{\text {int }}}{T}+\frac{1}{2}\right) / \sigma\right], m_{2}=\left[\left(\frac{t}{T}+\frac{1}{2}\right) / \sigma\right]+1$, and $[\cdot]$ denotes the integer part. This implies that for any $t>0$ and $\boldsymbol{x} \in S(t)$ the number of terms $m=m_{2}-m_{1}+1$ in the sum (21), and as such, the number of non-zero terms in the expansion (19), may not exceed $\left[\frac{T_{\text {int }}}{\sigma T}\right]+2$. Most important, this number $m$ does not increase as the time $t$ elapses, because the interval $T_{\text {int }}$ introduced in (20) depends only on the partition size $T$ for the sources, the geometry, the propagation speed, and the maximum speed of motion.

Let us now assume that each problem (18) is integrated individually on an appropriate domain of size $Z$, see formula (13), by means of a consistent and stable finite-difference scheme. For every given $j$, system (18) only needs to be integrated from $t_{0}^{(j)}=\left(\sigma j-\frac{1}{2}\right) T$ till $t_{2}^{(j)}=t_{0}^{(j)}+T_{\text {int }}$ because for all subsequent moments of time its solution on $S(t)$ will be equal to zero. Consequently, the following convergence estimates will hold for $t_{0}^{(j)} \leq t \leq t_{2}^{(j)}$ and $\boldsymbol{x} \in S(t)$ :

$$
\begin{aligned}
\left\|p^{(j)}(\boldsymbol{x}, t)-p_{h}^{(j)}(\boldsymbol{x}, t)\right\| & \leq K_{j} h^{\alpha}, \\
\left\|\boldsymbol{u}^{(j)}(\boldsymbol{x}, t)-\boldsymbol{u}_{h}^{(j)}(\boldsymbol{x}, t)\right\| & \leq K_{j} h^{\alpha},
\end{aligned}
$$

where $\alpha$ is the order of convergence, $h$ denotes the generic grid size, and the functions $p_{h}^{(j)}(\boldsymbol{x}, t)$ and $\boldsymbol{u}_{h}^{(j)}(\boldsymbol{x}, t)$ denote the discrete solution of system (18) for a given $j$. The constant $K_{j}$ on the right-hand side of each inequality (22) does not depend on $h$, but may depend on $q_{\mathrm{vol}}^{(j)}$ and $\boldsymbol{b}_{\mathrm{vol}}^{(j)}$, as well as on $T_{\text {int }}$.

We emphasize that the quantity $T_{\text {int }}$ does not depend on $j$. Moreover, it is natural to assume that the derivatives of the functions $q_{\mathrm{vol}}^{(j)}(\boldsymbol{x}, t)$ and $\boldsymbol{b}_{\mathrm{vol}}^{(j)}(\boldsymbol{x}, t)$ are 
uniformly bounded with respect to $j$. In this case, there will be a $j$-independent constant $K=K\left(q_{\mathrm{vol}}, \boldsymbol{b}_{\mathrm{vol}}, T_{\mathrm{int}}\right)$ such that $\forall j=0,1,2, \ldots: K_{j} \leq K$. Then, using representations (21) one can easily transform the individual convergence estimates (22) into the overall temporally uniform grid convergence estimate for $p$ and $\boldsymbol{u}$ that would hold for $t \geq 0$ and $\boldsymbol{x} \in S(t)$ :

$$
\begin{aligned}
\left\|p(\boldsymbol{x}, t)-p_{h}(\boldsymbol{x}, t)\right\| & \leq m K h^{\alpha}, \\
\left\|\boldsymbol{u}(\boldsymbol{x}, t)-\boldsymbol{u}_{h}(\boldsymbol{x}, t)\right\| & \leq m K h^{\alpha} .
\end{aligned}
$$

In formulae $(23), p_{h}(\boldsymbol{x}, t)=\sum_{j=m_{1}}^{m_{2}} p_{h}^{(j)}(\boldsymbol{x}, t)$ and $\boldsymbol{u}_{h}(\boldsymbol{x}, t)=\sum_{j=m_{1}}^{m_{2}} \boldsymbol{u}_{h}^{(j)}(\boldsymbol{x}, t)$. A detailed proof of this result for the wave equation can be found in [12].

In practical terms, the temporally uniform grid convergence guaranteed by estimates (23) means that accuracy of the numerical solution of system (10), if computed using lacunae, i.e., by solving a set of systems (18) and then employing representation (21), will not deteriorate even when system (10) is integrated over arbitrarily long time intervals. In other words, one should expect that there will be no long-time error buildup. This is, in fact, a key distinction between the foregoing lacunae-based algorithm and traditional time-marching techniques that may be applied to computing the unsteady acoustic fields.

Indeed, the phenomenon of error accumulation during long runs is well-known in the context of building computational methods for time-dependent problems. This issue has been recognized in the literature as an outstanding unresolved question in numerical PDEs for many years, since the first systematic convergence studies for discrete approximations have been conducted in the fifties. At the analysis stage, it manifests itself by the growth of the stability constants with time. If, for example, system (10) needs to be integrated over the interval $\left[0, T_{\text {final }}\right]$, then the stability constant of the scheme will, generally speaking, depend on $T_{\text {final }}: K=K\left(\cdot, T_{\text {final }}\right)$, and will actually increase with the increase of $T_{\text {final }}$. This is, of course, the exact same phenomenon as the dependence of $K_{j}$ on $T_{\text {int }}$ in formulae (22). The growth of the stability constants with $T_{\text {final }}$ is equivalent to non-uniformity of the grid convergence in time, and all conventional discrete approximations that can be and are used in modern numerical methods are known to suffer from this deficiency. In practice, this implies that any given approximation can be used for only a limited interval of time if the acceptable level of error is prescribed. To advance further in time with no loss of accuracy a finer approximation is needed from the very beginning, which obviously prompts the increase of the overall computational cost. The latter increase may quickly become prohibitive.

Using the language of wave physics, one can, e.g., attribute the long-term error buildup to either numerical dissipation, or dispersion (phase error), or both. But no matter what its actual mechanism is, it may result in an unacceptable 
loss of accuracy by the solution within a finite period of time.

The lacunae-based algorithm allows us to circumvent this difficulty due to the temporally uniform convergence (23). Moreover, if system (10) were to be integrated on the large interval $\left[0, T_{\text {final }}\right]$ using a straightforward time-marching algorithm, it would have also required a large domain in space, of the size roughly $2 c T_{\text {final }}$. This is typically not feasible. On the other hand, implementation of the lacunae-based algorithm allows us to perform the integration on the domain of a fixed and non-increasing size $Z$ determined by formula (13).

It is important to mention that smoothness plays a key role in the design of the lacunae-based algorithm. In particular, the function $\Theta(t)$ of $(15)$ that helps us build the partition of unity (14) has to be chosen sufficiently smooth so that the dependence of the stability constants $K_{j}$ on the properties of individual RHSs $q_{\mathrm{vol}}^{(j)}$ and $\boldsymbol{b}_{\mathrm{vol}}^{(j)}$, see (22), be not worse than that in the original scheme with non-partitioned source terms. In this paper, we leave out the detailed analysis that involves the quantitative smoothness characteristics, and instead refer the reader to our previous work [12].

Implementation of the lacunae-based algorithm now needs to be discussed. In theory, system (18) for every given $j=0,1,2, \ldots$ should be integrated on its own auxiliary region of size $Z$, see (13), centered around the domain $S\left(t_{0}^{(j)}\right)$, where $t_{0}^{(j)}=\left(\sigma j-\frac{1}{2}\right) T$ and the location of $S\left(t_{0}^{(j)}\right)$ is, in turn, determined by the reference point $\boldsymbol{x}_{0}\left(t_{0}^{(j)}\right)$, see formula (9). However, it is more convenient to consider periodic boundary conditions with the period $Z$ in all coordinate directions. In this case, motion (9) should be interpreted as the motion on a three-dimensional toroidal surface, and all spatial locations shall be converted to the periodic setting: $\boldsymbol{x} \mapsto \tilde{\boldsymbol{x}} \equiv\left(\tilde{x}_{1}, \tilde{x}_{2}, \tilde{x}_{3}\right)$, where $\tilde{x}_{i}=x_{i}-\left[\frac{x_{i}}{Z}\right] Z, i=$ $1,2,3$. In so doing, all systems (18) can basically be solved on one and the same domain with periodic boundary conditions, because it obviously does not matter where on the period the "initial" domain $S\left(t_{0}^{(j)}\right)$ is located for every $j=0,1,2, \ldots$ Moreover, while the most universal formulation would imply choosing the same period $Z$ for all the coordinates, in the case when motion (9) is characterized by a predominant direction, the periods in the directions orthogonal to that can be chosen smaller. For subsequent analysis in the current paper, the periodic setup will always be assumed.

Next, let us recast each formula (21) for the discrete case (subscript " $h$ ") in the form of a difference:

$$
\begin{array}{ccc} 
& p_{h}(\boldsymbol{x}, t)=\sum_{j=m_{1}}^{m_{2}} p_{h}^{(j)}(\boldsymbol{x}, t) & \boldsymbol{u}_{h}(\boldsymbol{x}, t)=\sum_{j=m_{1}}^{m_{2}} \boldsymbol{u}_{h}^{(j)}(\boldsymbol{x}, t) \\
=\sum_{j=0}^{m_{2}} p_{h}^{(j)}(\boldsymbol{x}, t)-\sum_{j=0}^{m_{1}-1} p_{h}^{(j)}(\boldsymbol{x}, t) & \text { and } & =\sum_{j=0}^{m_{2}} \boldsymbol{u}_{h}^{(j)}(\boldsymbol{x}, t)-\sum_{j=0}^{m_{1}-1} \boldsymbol{u}_{h}^{(j)}(\boldsymbol{x}, t)
\end{array}
$$


Existence of the upper limit $j=m_{2}$ in the summation (21) or (24) is due to the causality which is always a factor, and has nothing to do with the lacunae. Therefore, each minuend in formulae $(24), \sum_{j=0}^{m_{2}} p_{h}^{(j)}(\boldsymbol{x}, t)$ or $\sum_{j=0}^{m_{2}} \boldsymbol{u}_{h}^{(j)}(\boldsymbol{x}, t)$, could have simply been obtained by a straightforward time-marching of system (10) on the interval $[0, t)$ in the foregoing periodic setting, with absolutely no regard to either the partition (16) or split systems (18). The full quantity, $p_{h}(\boldsymbol{x}, t)$ or $\boldsymbol{u}_{h}(\boldsymbol{x}, t)$, cannot, of course, be obtained by only marching. To properly address the presence of the subtrahends $\sum_{j=0}^{m_{1}-1} p_{h}^{(j)}(\boldsymbol{x}, t)$ and $\sum_{j=0}^{m_{1}-1} \boldsymbol{u}_{h}^{(j)}(\boldsymbol{x}, t)$ in formulae (24), let us first symbolically write down the time-marching scheme that would apply to system (10), as well as to all systems (18):

$$
\begin{aligned}
& p_{h}(\boldsymbol{x}, t+\Delta t)=\mathcal{P}\left(p_{h}(\boldsymbol{x}, t), \boldsymbol{u}_{h}(\boldsymbol{x}, t), q_{\mathrm{vol}}(\boldsymbol{x}, t)\right), \\
& \boldsymbol{u}_{h}(\boldsymbol{x}, t+\Delta t)=\mathcal{U}\left(p_{h}(\boldsymbol{x}, t), \boldsymbol{u}_{h}(\boldsymbol{x}, t), \boldsymbol{b}_{\mathrm{vol}}(\boldsymbol{x}, t)\right) .
\end{aligned}
$$

Scheme (25) is chosen two-level explicit for simplicity only, this is by no means a limitation, and the analysis for multi-level schemes can be found in [12]. Consider now a particular moment of time $t$ that corresponds to the change in the lower summation limit in formulae (21), and accordingly (24), from $j=m_{1}$ to $j=m_{1}+1$, i.e., such $t$ that

$$
\underbrace{\left[\left(\frac{t+\Delta t-T_{\text {int }}}{T}+\frac{1}{2}\right) / \sigma\right]}_{m_{1}+1}=\underbrace{\left[\left(\frac{t-T_{\text {int }}}{T}+\frac{1}{2}\right) / \sigma\right]}_{m_{1}}+1 .
$$

Combining formulae (24) and (25), we will then have:

$$
\begin{aligned}
& p_{h}(\boldsymbol{x}, t+\Delta t)=\mathcal{P}\left(p_{h}(\boldsymbol{x}, t), \boldsymbol{u}_{h}(\boldsymbol{x}, t), q_{\mathrm{vol}}(\boldsymbol{x}, t)\right)-p_{h}^{\left(m_{1}\right)}(\boldsymbol{x}, t+\Delta t), \\
& \boldsymbol{u}_{h}(\boldsymbol{x}, t+\Delta t)=\mathcal{U}\left(p_{h}(\boldsymbol{x}, t), \boldsymbol{u}_{h}(\boldsymbol{x}, t), \boldsymbol{b}_{\mathrm{vol}}(\boldsymbol{x}, t)\right)-\boldsymbol{u}_{h}^{\left(m_{1}\right)}(\boldsymbol{x}, t+\Delta t) .
\end{aligned}
$$

In other words, when the current moment of time $t$ satisfies the "switch" condition (26), the terms $p_{h}^{\left(m_{1}\right)}(\boldsymbol{x}, t+\Delta t)$ and $\boldsymbol{u}_{h}^{\left(m_{1}\right)}(\boldsymbol{x}, t+\Delta t)$ need to be explicitly subtracted from the respective overall expressions, see (27), on top of the standard time-marching step as per (25). This basically amounts to the required change of the upper summation limit in both subtrahends of formulae (24) from $m_{1}-1$ to $m_{1}$. We will also assume hereafter that similarly to the original differential equations (10), the scheme (25) will satisfy the linear superposition principle. Then, the next time step after the one defined by formulae (27), i.e., the step $t+\Delta t \longmapsto t+2 \Delta t$, shall only be done by marching (25). Indeed, the subtracted quantities $p_{h}^{\left(m_{1}\right)}(\boldsymbol{x}, t+\Delta t)$ and $\boldsymbol{u}_{h}^{\left(m_{1}\right)}(\boldsymbol{x}, t+\Delta t)$ will carry over to all the steps that follow (27) due to the linearity. The genuine "unperturbed" marching can thus continue till the next switching moment $t$, i.e., till condition (26) is satisfied by $m_{1}+2$ and $m_{1}+1$. At this moment, the 
quantities $p_{h}^{\left(m_{1}+1\right)}(\boldsymbol{x}, t+\Delta t)$ and $\boldsymbol{u}_{h}^{\left(m_{1}+1\right)}(\boldsymbol{x}, t+\Delta t)$ will need to be subtracted, and then the procedure will cyclically repeat itself.

Thus, the lacunae-based algorithm can be implemented as a conventional timemarching procedure supplemented by repeated subtraction of the retarded terms. The subtraction moments are known up-front and separated from one another by equal time increments. The subtracted terms $p_{h}^{\left(m_{1}\right)}(\boldsymbol{x}, t+\Delta t)$ and $\boldsymbol{u}_{h}^{\left(m_{1}\right)}(\boldsymbol{x}, t+\Delta t)$ are legitimately called retarded because for a given moment of time $t$ that satisfies (26), they are generated by the RHSs $q_{\mathrm{vol}}^{\left(m_{1}\right)}$ and $\boldsymbol{b}_{\mathrm{vol}}^{\left(m_{1}\right)}$ that are active in the past, on the time interval $\left[t_{0}^{\left(m_{1}\right)}, t_{0}^{\left(m_{1}\right)}+T\right]=\left[\left(\sigma m_{1}-\right.\right.$ $\left.\left.\frac{1}{2}\right) T,\left(\sigma m_{1}+\frac{1}{2}\right) T\right]=\left[t-T_{\text {int }}, t-T_{\text {int }}+T\right]$. Of course, the actual subtracted quantities $p_{h}^{\left(m_{1}\right)}(\boldsymbol{x}, t+\Delta t)$ and $\boldsymbol{u}_{h}^{\left(m_{1}\right)}(\boldsymbol{x}, t+\Delta t)$ need to be re-computed for every $m_{1}$ independently of the primary time-marching procedure. This is done by means of the same scheme (25) applied to the corresponding system (18).

To conclude this section, we note that the original idea behind using the lacunae is to keep the number of terms in sums (21) or (24) fixed and nonincreasing, while still guaranteeing that the solution for $\boldsymbol{x} \in S(t)$ will be the same as if we integrated system (10) continuously starting from $t=0$. Besides, existence of the lower summation limit $j=m_{1}$ in (21) and (24), i.e., repeated subtraction of the retarded terms, serves an additional important purpose. It keeps the reflected waves from coming back into the domain $S(t)$ after the time interval $T_{\text {int }}$ has elapsed since the inception of any given component (16) of the RHS. Unless explicitly subtracted, these waves generated by the sources $q_{\mathrm{vol}}^{(j)}$ and $\boldsymbol{b}_{\mathrm{vol}}^{(j)}$ on the time interval $\left[t_{0}^{(j)}, t_{0}^{(j)}+T\right]$ for every given $j$, will start "contaminating" the solution on $S(t)$ right after the moment $t_{0}^{(j)}+T_{\text {int }}$.

\section{Lacunae-Based ABCs}

Suppose that the original formulation of the problem that we want to solve involves the entire space $\mathbb{R}^{3}$, but we are only interested to find a fragment of the overall solution defined on the domain $S(t)$. As in Section 4, the latter is supposed to have a fixed shape and finite size, but is allowed to move according to the law (9). While not making any specific assumptions regarding the nature of the phenomena/processes that are going on inside $S(t)$, we assume that outside $S(t)$, i.e., $\forall t>0$ and $\boldsymbol{x} \notin S(t)$, the appropriate model would be based on the homogeneous acoustics system:

$$
\begin{aligned}
\frac{1}{c^{2}} \frac{\partial p(\boldsymbol{x}, t)}{\partial t}+\rho_{0} \nabla \cdot \boldsymbol{u}(\boldsymbol{x}, t) & =0 \\
\rho_{0} \frac{\partial \boldsymbol{u}(\boldsymbol{x}, t)}{\partial t}+\nabla p(\boldsymbol{x}, t) & =\boldsymbol{O} .
\end{aligned}
$$


We assume that the overall problem, i.e., the interior one that we do not specify, combined with the exterior one, which is governed by system (28), is uniquely solvable on $\mathbb{R}^{3}$. In other words, our model may include some possibly complex phenomena confined to the bounded region $S(t)$ that manifest themselves by the acoustic sound in the far field. The objective is to actually solve the problem on the domain $S(t)$ using a numerical method, but truncate all of its exterior replacing it with the ABCs on the external boundary of $S(t)$. The ideal or exact ABCs would make the foregoing replacement equivalent, which means that the solution obtained this way would coincide on $S(t)$ with the corresponding fragment of the original infinite-domain solution. The latter, however, is not actually available because the far-field sound propagation governed by (28) cannot be computed directly at an acceptable cost.

From the viewpoint of an observer inside $S(t)$, the role of the ABCs at $\partial S(t)$ is only to guarantee that this boundary will behave exactly as if the domain $S(t)$ were surrounded by an infinite linear isotropic sound-conducting medium. In particular, the boundary $\partial S(t)$ may not reflect, fully or partially, any outgoing waves. Therefore, as far as the ABCs are concerned, we indeed do not have to be very specific regarding the nature of the problem inside $S(t)$, provided that the overall interior/exterior problem does have a unique solution.

The latter assumption is of central importance. However, justifying it for every particular formulation is beyond the scope of the current paper. For example, the overall problem may be linear, and therefore, uniquely solvable, such as acoustic scattering from a given solid inside $S(t)$. On the other hand, one may consider a substantially more complex model inside $S(t)$, such as the unsteady flow around a maneuvering aircraft. The flow linearizes in the far field thus reducing the original Euler's equations to system (28) at a distance from the aircraft. In this case, justifying the overall solvability theoretically is difficult at best. Besides, linearization in the far field is only an approximation that becomes more accurate the further away from the aircraft it is introduced. This is going to affect the final accuracy of the resulting ABCs. ${ }^{2}$ Hereafter, we will not be primarily concerned with either the validity of the linear model (28) outside $S(t)$, or with the overall solvability issue. We will rather focus on constructing the ABCs under these assumptions keeping in mind that they may need to be corroborated independently for each specific case.

There will be two stages in constructing the ABCs. First, an auxiliary problem will be formulated that will have the exact same solution outside $S(t)$ as the original combined problem does, but will be linear throughout the entire space $\mathbb{R}^{3}$ and will be driven by the specially derived source terms concentrated only inside $S(t)$. In other words, this problem will be of the type considered

$\overline{2}$ Our previous steady-state ABCs for external flows [22] were based on the far-field linearization and have still proven superior to other methods. 
in Section 4. Next, the auxiliary problem will be solved on a domain slightly larger than $S(t)$ using the lacunae-based methodology of Section 4, and its solution obtained right outside $S(t)$ will be used to supply the boundary conditions for the original interior problem solved inside $S(t)$. In practice, the two aforementioned stages will be meshed together so that both the interior problem and the auxiliary problem are time-marched concurrently. The entire algorithm will be implemented directly on the discrete level.

We note that the intention to use lacunae for solving the auxiliary problem imposes a certain restriction on the class of admissible formulations, because by doing so the acoustic far field is assumed vorticity free. It is also known, however, that for the linearized flows the vortical and acoustic modes essentially decouple. This suggests that the proposed methodology can potentially be used to set the ABCs for the acoustic part only, whereas for vorticity a different method may be employed (convection along entropy characteristics).

Let us define a subdomain $S_{\varepsilon}(t) \subset S(t)$ such that $\boldsymbol{x} \in S_{\varepsilon}(t)$ if and only if $\boldsymbol{x} \in S(t)$ and $\operatorname{dist}(\boldsymbol{x}, \partial S(t))>\varepsilon$, where $\varepsilon>0$, and introduce a multiplier function $\mu=\mu(\boldsymbol{x}, t)$ that is smooth across the entire space and $\forall t>0$ satisfies:

$$
\mu(\boldsymbol{x}, t) \equiv \begin{cases}0, & \boldsymbol{x} \in S_{\varepsilon}(t), \\ 1, & \boldsymbol{x} \notin S(t), \\ \in(0,1), & \boldsymbol{x} \in S(t) \backslash S_{\varepsilon}(t),\end{cases}
$$

The curvilinear strip $S(t) \backslash S_{\varepsilon}(t)$ of width $\epsilon$ adjacent to the boundary $\partial S(t)$ of the domain $S(t)$ from inside will hereafter be called the transition region.

Assume now that the solution to the combined interior/exterior problem is known on $\mathbb{R}^{3}$ for $t>0$. Clearly, it should satisfy $p(\boldsymbol{x}, 0)=0$ and $\boldsymbol{u}(\boldsymbol{x}, 0)=\boldsymbol{O}$ for $\boldsymbol{x} \in \mathbb{R}^{3} \backslash S(0)$. It is also important to realize that the unknown quantities inside and outside $S(t)$ do not necessarily have to be the same. For example, if the interior problem is that of the flow around an aircraft, then the unknowns inside $S(t)$ will be the actual flow variables, whereas the variables $p(\boldsymbol{x}, t)$ and $\boldsymbol{u}(\boldsymbol{x}, t)$ in system (28) that is used outside $S(t)$ are perturbations with respect to the corresponding background. We can, however, assume with no loss of generality that the definitions of the interior and exterior quantities are equivalent on the (narrow) transition region $S(t) \backslash S_{\varepsilon}(t)$. In the foregoing example with an aircraft, this assumption would imply that linearization in the transition region is still valid.

Having re-defined the interior solution in terms of the exterior quantities on $S(t) \backslash S_{\varepsilon}(t)$, we multiply the overall solution everywhere by $\mu(\boldsymbol{x}, t)$ of $(29)$ :

$$
\begin{aligned}
p(\boldsymbol{x}, t) & \longmapsto \mu(\boldsymbol{x}, t) p(\boldsymbol{x}, t) \equiv \hat{p}(\boldsymbol{x}, t), \\
\boldsymbol{u}(\boldsymbol{x}, t) & \longmapsto \mu(\boldsymbol{x}, t) \boldsymbol{u}(\boldsymbol{x}, t) \equiv \hat{\boldsymbol{u}}(\boldsymbol{x}, t) .
\end{aligned}
$$




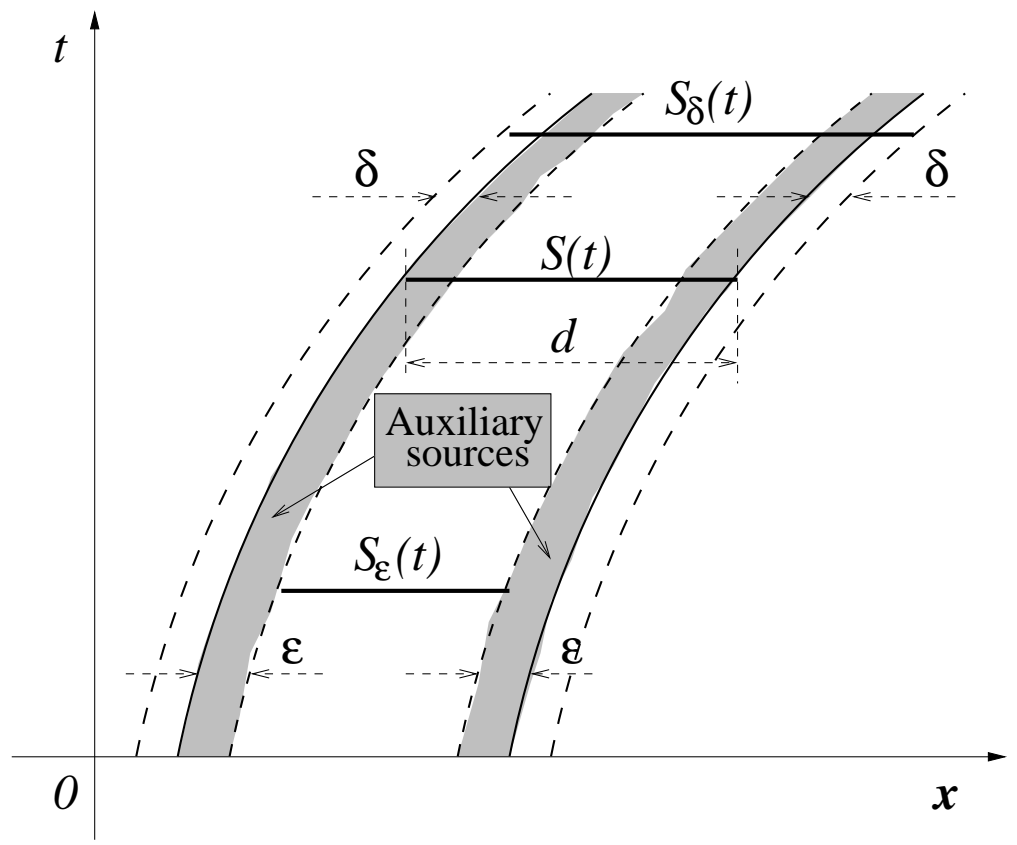

Fig. 1. Schematic geometry of the auxiliary sources region.

We emphasize that in order to obtain $\hat{p}(\boldsymbol{x}, t)$ and $\hat{\boldsymbol{u}}(\boldsymbol{x}, t)$, see (30), we do not need to know $p(\boldsymbol{x}, t)$ and $\boldsymbol{u}(\boldsymbol{x}, t)$ on $S_{\varepsilon}(t)$, because the multiplier $\mu$ is equal to zero there anyway. Moreover, multiplication (30) will not change any quantities on $\mathbb{R}^{3} \backslash S(t)$. With that in mind, we apply the differential operator from the left-hand side of system (28) to the modified solution $\hat{p}(\boldsymbol{x}, t), \hat{\boldsymbol{u}}(\boldsymbol{x}, t)$, see (30), and obtain:

$$
\begin{array}{r}
\frac{1}{c^{2}} \frac{\partial \hat{p}}{\partial t}+\rho_{0} \nabla \cdot \hat{\boldsymbol{u}}= \begin{cases}0, & \boldsymbol{x} \notin S(t) \backslash S_{\varepsilon}(t), \\
\rho_{0} \hat{q}_{\mathrm{vol}}, & \boldsymbol{x} \in S(t) \backslash S_{\varepsilon}(t),\end{cases} \\
\rho_{0} \frac{\partial \hat{\boldsymbol{u}}}{\partial t}+\nabla \hat{p}= \begin{cases}\boldsymbol{o}, & \boldsymbol{x} \notin S(t) \backslash S_{\varepsilon}(t), \\
\hat{\boldsymbol{b}}_{\mathrm{vol}}, & \boldsymbol{x} \in S(t) \backslash S_{\varepsilon}(t) .\end{cases}
\end{array}
$$

The result will indeed be zero on $S_{\varepsilon}(t)$, see (31), because $\mu(\boldsymbol{x}, t)=0$ for $\boldsymbol{x} \in$ $S_{\varepsilon}(t)$. The result is also zero on the entire exterior domain $\mathbb{R}^{3} \backslash S(t)$, see (31), because system (28) holds there, and the quantities $\hat{p}(\boldsymbol{x}, t)$ and $\hat{\boldsymbol{u}}(\boldsymbol{x}, t)$ coincide with $p(\boldsymbol{x}, t)$ and $\boldsymbol{u}(\boldsymbol{x}, t)$, respectively, on $\mathbb{R}^{3} \backslash S(t)$, as the second identity (29) combined with definitions (30) suggest. The result in (31) may differ from zero only in the transition region $S(t) \backslash S_{\varepsilon}(t)$, where we actually generate the RHSs $\hat{q}_{\mathrm{vol}}(\boldsymbol{x}, t)$ and $\hat{\boldsymbol{b}}_{\mathrm{vol}}(\boldsymbol{x}, t)$. Note, the smoothness of the original solution $p(\boldsymbol{x}, t)$, $\boldsymbol{u}(\boldsymbol{x}, t)$, as well as that of the multiplier $\mu(\boldsymbol{x}, t)$, guarantee that these new auxiliary sources $\hat{q}_{\mathrm{vol}}(\boldsymbol{x}, t)$ and $\hat{\boldsymbol{b}}_{\mathrm{vol}}(\boldsymbol{x}, t)$ will be smooth compactly supported functions. In Figure 1, we schematically depict the geometry of the region on which the auxiliary sources are defined.

Next, we substitute the auxiliary sources $\hat{q}_{\mathrm{vol}}(\boldsymbol{x}, t)$ and $\hat{\boldsymbol{b}}_{\mathrm{vol}}(\boldsymbol{x}, t)$ of $(31)$ into 
the RHS of system (10) and integrate the latter subject to the homogeneous initial conditions. Because of the overall regularity that we have assumed ahead of time, the solution of this new problem that we will further refer to as the auxiliary problem, will be unique and will coincide with the modified functions $\hat{p}(\boldsymbol{x}, t)$ and $\hat{\boldsymbol{u}}(\boldsymbol{x}, t)$ of $(30)$ on the entire space. This means that on $\mathbb{R}^{3} \backslash S(t)$ the solution to the auxiliary problem will coincide with the original exterior solution $p(\boldsymbol{x}, t), \boldsymbol{u}(\boldsymbol{x}, t)$. We emphasize that we do not need to explicitly know the original exterior solution on $\mathbb{R}^{3} \backslash S(t)$ in order to obtain the source terms $\hat{q}_{\mathrm{vol}}(\boldsymbol{x}, t)$ and $\hat{\boldsymbol{b}}_{\mathrm{vol}}(\boldsymbol{x}, t)$ that drive the auxiliary problem, we only need to know that it satisfies the homogeneous acoustics system (28).

Altogether, we have split the original problem into two: The linear auxiliary problem that needs to be solved on the entire space, and the interior problem on $S(t)$ that will be integrated with the external boundary data provided by the solution of the auxiliary problem. However, to apply the lacunae-based algorithm of Section 4 to the auxiliary problem, it may need to be modified. Namely, conservativeness of the auxiliary forcing $\hat{\boldsymbol{b}}_{\mathrm{vol}}$ of (31) must be maintained so that to guarantee existence of the lacunae in the auxiliary solutions, see Propositions 1 and 2. Let us assume that in the original combined problem the velocity potential does exist in the solutions of system (28) on $\mathbb{R}^{3} \backslash S(t)$. It is reasonable to think that it will exist on the transition region $S(t) \backslash S_{\epsilon}(t)$ as well, and that altogether the potential function $\varphi(\boldsymbol{x}, t)$ will be smooth. However, multiplication (30) may ruin the conservativeness. As such, we can first reconstruct the velocity potential $\varphi(\boldsymbol{x}, t)$ for $\boldsymbol{x} \in S(t) \backslash S_{\epsilon}(t)$ based on the computed interior quantities, multiply it by $\mu$, and only then obtain the modified velocity vector $\hat{\boldsymbol{u}}(\boldsymbol{x}, t)$, thus replacing (30) with the following steps:

$$
\begin{gathered}
p(\boldsymbol{x}, t) \longmapsto \mu(\boldsymbol{x}, t) p(\boldsymbol{x}, t) \equiv \hat{p}(\boldsymbol{x}, t), \\
\boldsymbol{u}(\boldsymbol{x}, t)=\nabla \varphi(\boldsymbol{x}, t), \quad \boldsymbol{x} \in S(t) \backslash S_{\epsilon}(t), \\
\varphi(\boldsymbol{x}, t) \longmapsto \mu(\boldsymbol{x}, t) \varphi(\boldsymbol{x}, t) \equiv \hat{\varphi}(\boldsymbol{x}, t), \\
\boldsymbol{u}(\boldsymbol{x}, t) \longmapsto \nabla \hat{\varphi}(\boldsymbol{x}, t) \equiv \hat{\boldsymbol{u}}(\boldsymbol{x}, t) .
\end{gathered}
$$

Subsequently, the modified functions $\hat{p}(\boldsymbol{x}, t)$ and $\hat{\boldsymbol{u}}(\boldsymbol{x}, t)$ of (32) are substituted into (31) to obtain the auxiliary RHSs.

The version of the algorithm that we actually implement in the current paper does include reconstruction of the potential in accordance with (32). We realize, of course, that this is by no means a must. In fact, all we need is a smooth extension of the exterior solution inwards that would transition to zero at a "depth" $\varepsilon$, and would also maintain conservativeness of the velocity field. There may be different ways of obtaining this extension, not necessarily based on applying $\mu$ to the interior solution on the transition region.

To set the discrete ABCs on the boundary $\partial S(t)$, we will need to apply the lacunae-based algorithm for solving the auxiliary problem (10), (31), (32) on 
a domain slightly larger than $\mathrm{S}(\mathrm{t})$. We take $\delta>0$ (to be specified later) and define $S_{\delta}(t)=\{(\boldsymbol{x}, t) \mid \operatorname{dist}(\boldsymbol{x}, S(t))<\delta\}$; clearly, $S_{\varepsilon}(t) \subset S(t) \subset S_{\delta}(t)$, see Figure 1. We also replace $\operatorname{diam} S(t)=d$ by $\operatorname{diam} S_{\delta}(t)=d+2 \delta$ in the formulae for the integration interval and auxiliary domain size [cf. (20), (13)]:

$$
T_{\mathrm{int}}=\frac{(d+2 \delta)+T(c+k)}{c-k} ; \quad Z=(d+2 \delta)+(c+k) T_{\mathrm{int}} .
$$

Next, assume that there is a space-time grid $\mathbb{N} \times \mathbb{T}$, on which a discrete approximation to the auxiliary problem is built. The spatial grid $\mathbb{N}$ consists of the nodes $\boldsymbol{n}$ in the three-dimensional space, whereas the temporal grid $\mathbb{T}$ is composed of the time levels $l=0,1,2, \ldots$ The grid $\mathbb{N}$ is actually introduced on the auxiliary domain of size $Z$ given by (33), and periodic boundary conditions are imposed. As no grid adaptation is needed, it is most convenient to simply use a uniform Cartesian grid. We also note that the original problem solved inside $S(t)$ does not have to be approximated on the same grid. In the most general situation, we will have different grids for the interior problem and for the exterior/auxiliary problem. Then, in the transition region $S(t) \backslash S_{\varepsilon}(t)$, where the definitions of the unknown quantities for both problems are equivalent, we may need to employ a chimera-type grid strategy, i.e., interpolate in-between the overlapping grids. For the analysis in the current paper, however, we will simply assume that the quantities from the interior solution are already available on the grid sub-domain $\{\mathbb{N} \times \mathbb{T}\} \cap\left\{S(t) \backslash S_{\varepsilon}(t)\right\}$.

Let us denote by $\mathbb{N}^{l}, l=0,1,2, \ldots$, the corresponding time levels of the grid $\mathbb{N} \times \mathbb{T}$, and by $\mathbb{S}_{n}^{l}$ the stencil of the scheme associated with the node $(\boldsymbol{n}, l) \in \mathbb{N} \times \mathbb{T}$. For simplicity, we will assume that the auxiliary problem (10), (31), (32) is time-marched by an explicit scheme, and that the node $(\boldsymbol{n}, l) \in \mathbb{S}_{n}^{l}$ is the actual upper-level node on the $q+1$-level stencil, i.e., $\mathbb{S}_{n}^{l} \cap\{\mathbb{N} \times \mathbb{T}\} \subset$ $\left\{\mathbb{N}^{l} \cup \mathbb{N}^{l-1} \cup \ldots \cup \mathbb{N}^{l-q}\right\}$ and $\mathbb{S}_{n}^{l} \cap \mathbb{N}^{l}=(\boldsymbol{n}, l)$. In Section 4 , we have assumed $q=1$, see formula (25). Denote by $\mathbb{N}_{+}^{l}$ the sub-grid of $\mathbb{N}^{l}$ that belongs to the interior domain, i.e., $\mathbb{N}_{+}^{l}=\mathbb{N}^{l} \cap S\left(t^{l}\right)$, where $t^{l}$ is the $l$-th time level in actual units ("seconds"), in the simplest case $t^{l}=l \Delta t$. Then, introduce the sum of the interior sub-grids for all time levels: $\mathbb{N}_{+}=\mathbb{N}_{+}^{0} \cup \mathbb{N}_{+}^{1} \cup \mathbb{N}_{+}^{2} \cup \ldots \subset \mathbb{N} \times \mathbb{T}$. Finally, consider a somewhat larger sub-grid of $\mathbb{N} \times \mathbb{T}: \quad \tilde{\mathbb{N}}_{+}=\underset{(n, l) \in \mathbb{N}_{+}}{\cup} \mathbb{S}_{n}^{l}$, which is simply a composition of all the stencils $\mathbb{S}_{n}^{l}$ obtained when the upperlevel node $(\boldsymbol{n}, l)$ sweeps the grid $\mathbb{N}_{+} ;$clearly, $\mathbb{N}_{+} \subset \tilde{\mathbb{N}}_{+}$. The part of the grid $\tilde{\mathbb{N}}_{+}$that does not belong to $\mathbb{N}_{+}$is called the grid boundary and is denoted $\gamma=\tilde{\mathbb{N}}_{+} \backslash \mathbb{N}_{+}$. We will require that the domain $S_{\delta}\left(t^{l}\right)$ be chosen so that on every time level $t^{l}, l=0,1,2, \ldots$, all of the grid $\tilde{\mathbb{N}}_{+}^{l}$ belong to this domain: $\tilde{\mathbb{N}}_{+}^{l} \subset S_{\delta}\left(t^{l}\right)$; equivalently, we may require that $\gamma^{l} \subset \mathbb{S}_{\delta}\left(t^{l}\right)$. We note that the grid boundary $\gamma$ is a narrow fringe of grid nodes that follows the geometry of $\partial S(t)$. Therefore, the parameter $\delta$ may be chosen small, on the order of a few grid sizes depending on the specific structure of $\mathbb{S}_{n}^{l}$. We also note that the way we have introduced the grid boundary $\gamma$ is actually a simplified interpretation 
of the rigorous general construction that is a part of the definition of discrete Calderon's potentials and boundary projection operators, the latter are used in [14] as a universal apparatus for setting the ABCs.

We will now describe one time step of the combined time-marching algorithm that involves the lacunae-based ABCs. We will assume that all time steps are identical and as such, will provide an inductive description of the algorithm.

Suppose that we have obtained the solution for up to a given time level $l$. This means that the solution is known not only on the interior domain, but also on the grid boundary - at the levels $\gamma^{l}, \ldots, \gamma^{l-q}$ that are immediately needed for advancing the next time step, as well as at all the preceding levels. In particular, one may think about starting the computation from the known (homogeneous) initial conditions. First, we make one interior time step and obtain the discrete solution everywhere inside including the transition region, i.e., the grid area $\mathbb{N}^{l+1} \cap\left\{S\left(t^{l+1}\right) \backslash S_{\varepsilon}\left(t^{l+1}\right)\right\}$, where the solution is assumed to be defined in terms of the exterior acoustic quantities $p(\boldsymbol{x}, t)$ and $\boldsymbol{u}(\boldsymbol{x}, t)$. Then, we perform the modification (32) in the discrete framework, which involves reconstruction of the velocity potential on the grid in the transition region $S\left(t^{l+1}\right) \backslash S_{\varepsilon}\left(t^{l+1}\right)$. A straightforward approach to that is contour integration along the grid lines; it has proven quite robust in our simulations, see Section 6, taking in to account that the potential only needs to be reconstructed on a narrow near-boundary strip. Having gotten the modified quantities (32) on the grid sub-domain $\mathbb{N}^{l+1} \cap\left\{S\left(t^{l+1}\right) \backslash S_{\varepsilon}\left(t^{l+1}\right)\right\}$, we apply the discrete version of (31) and obtain one more time level of the discrete auxiliary RHSs. If the scheme written on the stencil $\mathbb{S}_{n}^{l}$ approximates system (10) with the design accuracy at some node $\left(\boldsymbol{n}-\boldsymbol{n}_{0}, l-l_{0}\right) \in \mathbb{S}_{n}^{l}$ (clearly, $\left.l_{0} \leq q\right)$, then the discrete RHSs should be referred to this same node $\left(\boldsymbol{n}-\boldsymbol{n}_{0}, l-l_{0}\right)$ and as such, advancing the interior solution till $(l+1)$ would mean building the auxiliary RHSs till $\left(l+1-l_{0}\right)$. Next, we make one time step for the auxiliary problem with these newly updated RHSs, and obtain its solution on $\mathbb{N}^{l+1} \cap S_{\delta}\left(t^{l+1}\right)$. Since we have chosen $\delta>0$ so that $\tilde{\mathbb{N}}_{+}^{l+1} \subset S_{\delta}\left(t^{l+1}\right)$, we determine that the solution to the auxiliary problem will, in particular, be available on $\gamma^{l+1}$. This concludes one full time step, because once we know the solution on all time levels up to $(l+1)$ everywhere including the grid boundary, we can advance the next interior time step, etc.

The lacunae-based methodology for solving the auxiliary problem includes cyclic subtractions (27) of the retarded terms on top of the straightforward time-marching. It is important to realize that once a particular component has been subtracted, there will never be a need to analyze/incorporate it again in the course of computation. In other words, the subtracted terms are completely disregarded from the moment of subtraction further on. Therefore, the corresponding partition elements (16) of the auxiliary RHSs can be disregarded as well. Consequently, even when integrating over arbitrarily long 
time intervals, we only need to keep a finite amount of the past information, namely, the auxiliary RHSs (31) defined on the interval of duration $T_{\text {int }}$, see (33), that immediately precedes the current moment of time. This makes the extent of temporal nonlocality of the proposed ABCs fixed and limited.

The proposed ABCs guarantee that the external artificial boundary be completely transparent for all the outgoing waves. Indeed, they simply allow these waves to propagate beyond the boundary and then prevent reflections from re-entering the domain by eliminating the retarded components of the solution in a timely fashion. The ABCs-related computer expenses per unit time or per time step remain fixed and non-growing, which is accounted for by the lacunae-based integration. For explicit schemes, the operation count is proportional to the number of auxiliary grid nodes. The overall actual cost is, of course, higher than it would have been if the integration was performed on the domain $S(t)$ only, because the auxiliary domain is larger. The relation between the sizes is given by (33), and it also roughly indicates what the ratio of the work may be. However, we obviously cannot integrate on $S(t)$ alone, without boundary conditions. In this perspective, the overall performance assessment must include the increase of the cost that should be "weighted against" other characteristics such as accuracy of the boundary treatment and the range of problems that can be analyzed.

\section{Numerical Demonstrations}

For our numerical simulations, we assume axial symmetry and employ the $(r, z)$ cylindrical coordinates to account for the important three-dimensional effects using a two-dimensional spatial geometry. Let $u=u(r, z, t)$ and $w=w(r, z, t)$ be the radial and axial components of the acoustic velocity, respectively, and let $p=p(r, z, t)$ still denote the acoustic pressure. Let us also assume that $\rho_{0}=1$. Then, system (10) becomes:

$$
\begin{aligned}
\frac{1}{c^{2}} \frac{\partial p}{\partial t}+\frac{1}{r} \frac{\partial(r u)}{\partial r}+\frac{\partial w}{\partial z} & =q(r, z, t), \\
\frac{\partial u}{\partial t}+\frac{\partial p}{\partial r} & =b_{r}(r, z, t), \\
\frac{\partial w}{\partial t}+\frac{\partial p}{\partial z} & =b_{z}(r, z, t) .
\end{aligned}
$$

On the axis $r=0$ system (34a) changes. Namely, all the quantities involved must be continuous and bounded. Then, for the pressure, which is a scalar quantity, the axial symmetry (independence on the polar angle) implies: $\left.\frac{\partial p}{\partial r}\right|_{r=0}=0$. For the velocity, which is a vector quantity, we obtain $u(0, z, t)=0$ and $\left.\frac{\partial w}{\partial r}\right|_{r=0}=0$. Next, using Taylor's expansion for $r \ll 1$, 
we have: $u(r, \cdot)=u^{\prime}(0, \cdot) r+o(r)$ and consequently, $\frac{1}{r} \frac{\partial(r u)}{\partial r}=\frac{1}{r} \frac{\partial\left(r^{2} u^{\prime}(0, \cdot)\right)}{\partial r}+o(1)$, which means that $\left.\frac{1}{r} \frac{\partial(r u)}{\partial r}\right|_{r=0}=\left.2 \frac{\partial u}{\partial r}\right|_{r=0}$. Therefore, for $r=0$ system (34a) transforms into the system of two equations:

$$
\begin{aligned}
\frac{1}{c^{2}} \frac{\partial p}{\partial t}+2 \frac{\partial u}{\partial r}+\frac{\partial w}{\partial z} & =q(0, z, t), \\
\frac{\partial w}{\partial t}+\frac{\partial p}{\partial z} & =b_{z}(0, z, t),
\end{aligned}
$$

and the radial momentum equation degenerates for $r=0$.

The domain $S(t)$ will be a ball of fixed diameter $d$ centered on the $z$ axis, with the given axial velocity and coordinate of the center [cf. formulae (9)]:

$$
w_{0}=w_{0}(t), \quad z_{0}=z_{0}(t)=\int_{0}^{t} w_{0}(\tau) d \tau .
$$

Obviously, as the motion (35) is aligned with the axis $r=0$, it does not break the axial symmetry. We take the diameter $d=1.8$ and the speed of sound $c=1$; the functions $w_{0}(t)$, and $z_{0}(t)$ of $(35)$ will be specified later.

The auxiliary domain is a rectangle $[0, R] \times[-Z / 2, Z / 2]$ of variables $(r, z)$, with the actual sizes $R=\pi$ and $Z=2 \pi$. The boundary conditions for all variables are periodic with the period $Z$ of (33) in the $z$ direction:

$$
\{p, u, w\}(r, z \pm Z, t)=\{p, u, w\}(r, z, t) .
$$

In the radial direction, the boundary conditions cannot be periodic because of the geometry/symmetry considerations. At $r=0$, there is no need for the boundary conditions at all; instead, we have system $(34 \mathrm{~b})$ and $u(0, z, t)=0$. At $r=R$, the boundary conditions need to be provided for the homogeneous counterpart of system (34a), and we set:

$$
p(R, z, t)=0,\left.\quad \frac{\partial(r u)}{\partial r}\right|_{r=R}=0, \quad w(R, z, t)=0 .
$$

We note that the reflecting properties of boundary conditions (36a) and (36b) are basically immaterial for reconstructing the infinite-domain solution on $S(t)$, as long as the geometric restrictions discussed in the beginning of Section 4 are honored. We only need to make sure that the auxiliary problem is uniquely solvable, which boundary conditions (36a), (36b) do provide for. We leave out a detailed justification of the latter statement, only mention that the homogeneous Robin boundary condition (36b) for $u$ follows from the homogeneous Dirichlet boundary conditions (36b) for $p$ and $w$ combined with the homogeneous counterpart of the continuity equation in system (34a).

The acoustic test solution that we will be validating our numerical method on is supposed to have a conservative velocity field. Therefore, it will be con- 
venient to first construct the test solution for the potential, and then, by differentiating it, obtain the acoustic quantities, see (6). The latter will subsequently be reconstructed by integrating numerically the acoustics system, and continuous and discrete quantities will be compared against one another.

According to Proposition 1, velocity potential must satisfy the wave equation. For the moment, let us assume that this wave equation is driven by a moving point source with the amplitude $\chi=\chi(t)$ [cf. equation (1a)]:

$$
\frac{1}{c^{2}} \frac{\partial^{2} \varphi}{\partial t^{2}}-\Delta \varphi=\chi(t) \delta\left(\boldsymbol{x}-\boldsymbol{x}_{0}(t)\right) \equiv f(\boldsymbol{x}, t)
$$

Solution of equation (37) can be obtained as convolution with the fundamental solution of the wave equation,

$$
\mathcal{E}=\frac{H(t)}{4 \pi} \frac{\delta(|\boldsymbol{x}|-c t)}{t}
$$

see, e.g., [15], where $H(t)$ is the Heaviside function, and $\delta(|\boldsymbol{x}|-c t)$ is a single layer of unit magnitude on the expanding sphere of radius $c t$ [cf. formula (2)]:

$$
\begin{aligned}
\varphi=\mathcal{E} * f & =\frac{1}{4 \pi} \int_{0}^{\infty} d \tau \int_{\mathbb{R}^{3}} \frac{\delta(|\boldsymbol{x}-\boldsymbol{\xi}|-c(t-\tau))}{t-\tau} \chi(\tau) \delta\left(\boldsymbol{\xi}-\boldsymbol{x}_{0}(\tau)\right) d \boldsymbol{\xi} \\
& =\frac{1}{4 \pi} \int_{0}^{\infty} \frac{\delta\left(\left|\boldsymbol{x}-\boldsymbol{x}_{0}(\tau)\right|-c(t-\tau)\right)}{t-\tau} \chi(\tau) d \tau \\
& =\frac{1}{4 \pi} \int_{|\boldsymbol{x}|}^{\infty} \frac{\left|\boldsymbol{x}-\boldsymbol{x}_{0}(\tau)\right| \delta(\nu-c t)}{\left(c\left|\boldsymbol{x}-\boldsymbol{x}_{0}(\tau)\right|-\left\langle\boldsymbol{x}-\boldsymbol{x}_{0}(\tau), \boldsymbol{u}_{0}(\tau)\right\rangle\right)(t-\tau)} \chi(\tau) d \nu \\
& =\left.\frac{1}{4 \pi} \frac{c \chi(\tau)}{c\left|\boldsymbol{x}-\boldsymbol{x}_{0}(\tau)\right|-\left\langle\boldsymbol{x}-\boldsymbol{x}_{0}(\tau), \boldsymbol{u}_{0}(\tau)\right\rangle}\right|_{\nu=c t} .
\end{aligned}
$$

In formula $(38),\langle\cdot, \cdot\rangle$ denotes the dot product. For the integration, we have introduced a new variable $\nu=\left|\boldsymbol{x}-\boldsymbol{x}_{0}(\tau)\right|+c \tau$ as done in [23, Chapter 7]. Evaluating the last expression in (38) for $\nu=c t$ requires solving equation

$$
\left|\boldsymbol{x}-\boldsymbol{x}_{0}(\tau)\right|+c \tau=c t
$$

with respect to $\tau$. Solution of equation (39) determines the retarded moment of time, at which the trajectory of the point source intersects the lower portion of the characteristic cone with the vertex $(\boldsymbol{x}, t)$. For the case of a straightforward uniform motion, equation (39) is quadratic, and its solution $\tau$, once substituted in (38), yields $\varphi(\boldsymbol{x}, t)$ that can also be obtained via the Lorentz transform, as done in $[12,13]$. In general however, one should not expect to be able to solve the nonlinear equation (39) in the closed form.

Let us now take into account the cylindrical symmetry and straightforward 
motion (35). Then, formulae (38) and (39) reduce to

$$
\varphi(r, z, t)=\left.\frac{1}{4 \pi} \frac{c \chi(\tau)}{c \sqrt{r^{2}+\left(z-z_{0}(\tau)\right)^{2}}-\left(z-z_{0}(\tau)\right) w_{0}(\tau)}\right|_{\nu=c t}
$$

and

$$
\sqrt{r^{2}+\left(z-z_{0}(\tau)\right)^{2}}+c \tau=c t
$$

respectively. Assuming that $k_{1} \leq w_{0}(t) \leq k_{2}$, where $k_{1}$ and $k_{2}$ are known, we solve equation (41) by Newton's method with the initial guess $\tau_{0}$ given by the solution of the quadratic equation $r^{2}+\left(z-\frac{1}{2}\left(k_{1}+k_{2}\right) \tau_{0}\right)^{2}=c\left(t-\tau_{0}\right)^{2}$ that satisfies $\tau_{0}<t$. The actual law of motion that we specify is [cf. formulae (35)]:

$$
z_{0}(t)=\left[\frac{t}{10}\right]+\frac{1}{2}\left(1+\frac{15}{8} s-\frac{5}{4} s^{3}+\frac{3}{8} s^{5}\right), \quad s=2\left(\left\{\frac{t}{10}\right\}-\frac{1}{2}\right),
$$

where $[\cdot]$ denotes the integer part, as before, and $\{\cdot\}$ denotes the fractional part. The motion (42) basically consists of repeated acceleration/deceleration cycles of duration 10, so that during each cycle the source travels a total distance of 1 along the $z$ axis. Both the velocity $w_{0}(t)=z_{0}^{\prime}(t)$ and the acceleration $a_{0}(t)=z_{0}^{\prime \prime}(t)$ determined by (42) are continuous functions of time, and $0=k_{1} \leq w_{0}(t) \leq k_{2}=0.1875$, i.e., the subsonic condition is met.

Solution (40) for the potential is singular, and cannot be used directly to derive the acoustic quantities needed for testing the numerical procedure. To remove the singularity, we introduce a new smooth function $G=G(\tilde{r})$ of the variable $\tilde{r}=\tilde{r}(r, z, t) \equiv \sqrt{r^{2}+\left(z-z_{0}(t)\right)^{2}}$ such that $G(\tilde{r}) \equiv 1$ for $\tilde{r} \geq \frac{\kappa d}{2}$, where $\kappa<1$, and also such that at least $G(0)=G^{\prime}(0)=G^{\prime \prime}(0)=G^{\prime \prime \prime}(0)=0$. Then, the new function $\varphi(r, z, t) \cdot G(\tilde{r})$ is continuous and bounded everywhere. By differentiating it, we define the components of the reference acoustic solution [cf. formulae (6)]:

$$
\begin{aligned}
p(r, z, t) & =-\frac{\partial}{\partial t}(\varphi(r, z, t) \cdot G(\tilde{r})), \\
u(r, z, t) & =\frac{\partial}{\partial r}(\varphi(r, z, t) \cdot G(\tilde{r})), \\
w(r, z, t) & =\frac{\partial}{\partial z}(\varphi(r, z, t) \cdot G(\tilde{r})),
\end{aligned}
$$

which are regular functions as well. Note that $\varphi$ of (40) is a retarded potential; therefore, differentiation (43) involves implicit differentiation of $\tau$ via (41). At the same time, the definition of $G(\tilde{r})$ does not involve any retardation.

The variables $p, u$, and $w$ of (43) satisfy the homogeneous version of the acoustics system (34a) for $\tilde{r}>\frac{\kappa d}{2}$, where we have taken $\kappa=0.8$. Inside the smaller ball, i.e., for $\tilde{r} \leq \frac{\kappa d}{2}$, substitution of $p, u$, and $w$ of (43) into the left-hand 
side of system (34a) will produce the corresponding source terms $q, b_{r}$, and $b_{z}$. Note, when removing the singularity of the solution we have required that sufficiently many derivatives of $G$ be equal to zero at $\tilde{r}=0$; consequently, the RHSs due to the quantities (43) substituted into (34a) will have no singularities either. Altogether, we have therefore obtained a reference acoustic solution, which is regular everywhere, and which can be said to be generated by the sources concentrated inside the moving domain $S(t)$. Outside $S(t)$, our reference solution is basically a system of unsteady acoustic waves radiated by and propagating away from a moving source. We are going to reconstruct this solution numerically on the domain $S(t)$, and set the discrete lacunae-based ABCs on its outer boundary $\partial S(t)$ according to the methodology of Section 5.

The acoustics system (34a) is approximated numerically on the Cartesian grid:

$$
\begin{gathered}
r_{i}=i \Delta r, \quad \Delta r=R / N_{r}, \quad i=0,1, \ldots, N_{r}, \\
z_{j}=j \Delta z, \quad \Delta z=Z / 2 N_{z}, \quad j=0, \pm 1, \ldots, \pm N_{z},
\end{gathered}
$$

using a second-order staggered finite-difference scheme:

$$
\begin{aligned}
\frac{1}{c^{2}} \frac{p_{i+\frac{1}{2}, j}^{l+1}-p_{i+\frac{1}{2}, j}^{l}}{\Delta t}+\frac{1}{r_{i+\frac{1}{2}}} \frac{r_{i+1} u_{i+1, j}^{l+\frac{1}{2}}-r_{i} u_{i, j}^{l+\frac{1}{2}}}{\Delta r} & + \\
\frac{w_{i+\frac{1}{2}, j+\frac{1}{2}}^{l+\frac{1}{2}}-w_{i+\frac{1}{2}, j-\frac{1}{2}}^{l+\frac{1}{2}}}{\Delta z} & =q_{i+\frac{1}{2}, j}^{l+\frac{1}{2}}, \\
\frac{u_{i, j}^{l+\frac{1}{2}}-u_{i, j}^{l-\frac{1}{2}}}{\Delta t}+\frac{p_{i+\frac{1}{2}, j}^{l}-p_{i-\frac{1}{2}, j}^{l}}{\Delta r} & =b_{r_{i, j}}^{l}, \\
\frac{w_{i+\frac{1}{2}, j+\frac{1}{2}}^{l+\frac{1}{2}}-w_{i+\frac{1}{2}, j+\frac{1}{2}}^{l-\frac{1}{2}}}{\Delta t}+\frac{p_{i+\frac{1}{2}, j+1}^{l}-p_{i+\frac{1}{2}, j}^{l}}{\Delta z} & =b_{z_{i+\frac{1}{2}, j+\frac{1}{2}}^{l}}^{l}
\end{aligned}
$$

Scheme (45a) can be written for $i>0$, i.e., away from the axis of symmetry. For $i=0$, we set $u_{0, j}^{l+\frac{1}{2}}=0$ and approximate system (34b), which yields:

$$
\begin{aligned}
& \frac{1}{c^{2}} \frac{p_{\frac{1}{2}, j}^{l+1}-p_{\frac{1}{2}, j}^{l}}{\Delta t}+\frac{2}{\Delta r} u_{i, j}^{l+\frac{1}{2}}+\frac{w_{\frac{1}{2}, j+\frac{1}{2}}^{l+\frac{1}{2}}-w_{\frac{1}{2}, j-\frac{1}{2}}^{l+\frac{1}{2}}}{\Delta z}=q_{\frac{1}{2}, j}^{l+\frac{1}{2}}, \\
& \frac{w_{\frac{1}{2}, j+\frac{1}{2}}^{l+\frac{1}{2}}-w_{\frac{1}{2}, j+\frac{1}{2}}^{l-\frac{1}{2}}}{\Delta t}+\frac{p_{\frac{1}{2}, j+1}^{l}-p_{\frac{1}{2}, j}^{l}}{\Delta z}=b_{z \frac{1}{2}, j+\frac{1}{2}}^{l} .
\end{aligned}
$$

Scheme (45a) is very similar to the well-known Yee scheme that was originally introduced for solving the Maxwell equations, see [24]. Periodic boundary conditions (36a) are re-written on the grid as follows:

$$
p_{i+\frac{1}{2}, N_{z}}^{l}=p_{i+\frac{1}{2},-N_{z}}^{l}, \quad u_{i, N_{z}}^{l+\frac{1}{2}}=u_{i,-N_{z}}^{l+\frac{1}{2}}, \quad w_{i+\frac{1}{2}, N_{z}+\frac{1}{2}}^{l+\frac{1}{2}}=w_{i+\frac{1}{2},-N_{z}+\frac{1}{2}}^{l+\frac{1}{1}},
$$


whereas boundary conditions (36b) are discretized as:

$$
p_{N_{r}-\frac{1}{2}, j}^{l}=0, \quad \frac{r_{N_{r}} u_{N_{r}, j}^{l+\frac{1}{2}}-r_{N_{r}-1} u_{N_{r}-1, j}^{l+\frac{1}{2}}}{\Delta r}=0, \quad w_{N_{r}-\frac{1}{2}, j+\frac{1}{2}}^{l+\frac{1}{2}}=0 .
$$

We have used three successively more fine square cell grids, $\Delta r=\Delta z, N_{r} \times$ $2 N_{z}=64 \times 128,128 \times 256$, and $256 \times 512$. The Courant stability constraint was applied when selecting the time step for the explicit scheme (45a), (45b). The grid boundary $\gamma$ was built as outlined in Section 6, taking into account that scheme (45a), (45b) is staggered. The latter implies that we actually have three different stencils for updating the pressure and two velocity components that are shifted with respect to one another. Of course, the grid boundary is constructed concurrently with the actual time-marching. The parameter $\delta$ that is needed to accommodate the width of the grid boundary $\gamma$ was taken $\delta=$ $\frac{3}{2} \Delta r$. The functions $\Theta(t), \mu(\boldsymbol{x}, t)$, and $G(\tilde{r})$ introduced in order to guarantee smoothness at all the stages of the derivation, are constructed as piece-wise polynomials with four continuous derivatives everywhere. In so doing, the multiplier $\mu(\boldsymbol{x}, t)$, see $(29)$, is also built as a function of $\tilde{r}$ only. The varying amplitude $\chi$, see (40), was chosen in the form of a harmonic oscillation with the frequency three times that of the motion cycles (42). The width $\varepsilon$ of the transition region $S(t) \backslash S_{\varepsilon}(t)$, see Figure 1, varied to demonstrate different aspects of the algorithm performance. The parameter $\sigma$ of (14), (15) was chosen $\sigma=\frac{3}{4}$. The actual temporal thickness $T$ of each partition element was calculated "backwards" from (33), considering that the domain size, the maximum motion speed, see (42), and the period $Z$ are known. When marching the auxiliary problem, retarded components of the solution are subtracted according to (27). Each subtracted component is recomputed as solution to the corresponding problem (18). For a given $j$, this problem is inhomogeneous on the interval $\left[t_{0}^{(j)}, t_{1}^{(j)}\right] \equiv\left[\left(\sigma j-\frac{1}{2}\right) T,\left(\sigma j+\frac{1}{2}\right) T\right]$, and then it remains homogeneous on the interval $\left[t_{1}^{(j)}, t_{2}^{(j)}\right] \equiv\left[\left(\sigma j+\frac{1}{2}\right) T,\left(\sigma j-\frac{1}{2}\right) T+T_{\text {int }}\right]$. Therefore, we first explicitly time-march this system on its interval of inhomogeneity. Then, we do the FFT of the solution in the $z$ direction and expansion with respect to the corresponding eigenfunctions (evaluated numerically) in the $r$ direction, which allows us to advance the homogeneous solution further till $t_{2}^{(j)}$ by simply raising the resulting amplifications factors to the corresponding powers. We note that in so doing scheme (45a) effectively gets split into three discrete wave equations for the respective unknown quantities, because in the cylindrical geometry different transforms along $r$ are needed for different variables. This is the only instance when reduction to a set of independent wave equations is employed; and it is only necessitated by a particular choice of the coordinate system. If a genuine three-dimensional computation were conducted on a Cartesian grid, the unsplit system could have been used all along.

In Figure 2 we present error for the acoustic pressure as it depends on time on 


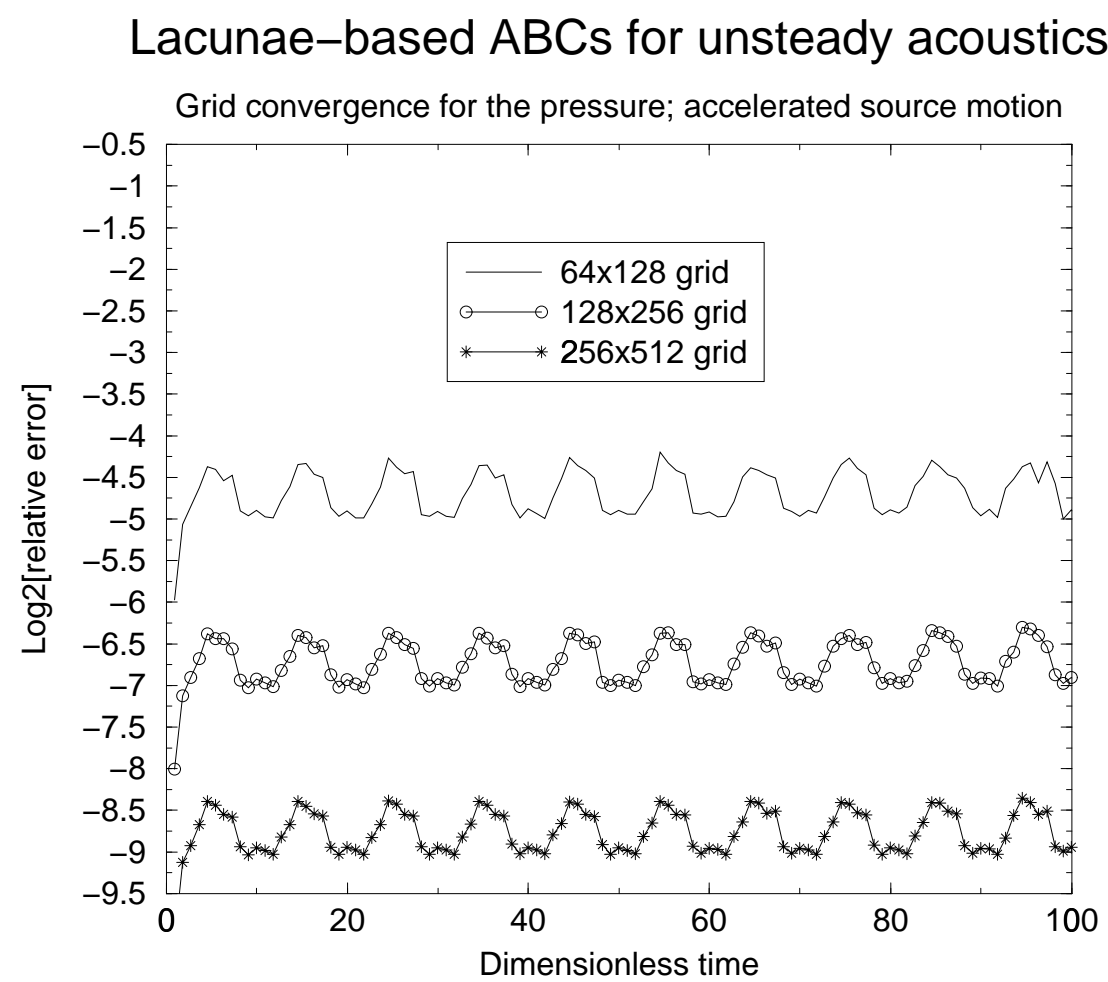

Fig. 2. Grid convergence study with lacunae-based ABCs, $\varepsilon=10 \Delta r$.

all three grids that we have employed. The total integration time was $100 \frac{d}{c}$, i.e., one hundred times the interval required for the waves to cross the domain. The error was evaluated in the maximum norm on the domain $S(t)$. We see that the algorithm provides for no long-term error buildup, and also that it displays the design second-order grid convergence. The oscillations in the error profiles on Figure 2 have the period of 10 time units, and apparently follow the acceleration/deceleration cycles of the source motion. Error profiles for the velocity components $u$ and $w$ look similarly and we do not present them.

We should mention that the error curves on Figure 2 were obtained for a relatively wide transition region $S(t) \backslash S_{\varepsilon}(t)$, on the order of ten grid cell sizes. Even though the actual width of this region decreases with the refinement of the grid, the issue of how the width $\varepsilon$ affects the algorithm performance deserves to be thoroughly addressed. From the numerical standpoint, the width of the transition region determines how well the smooth function $\mu(\boldsymbol{x}, t)$ is resolved on the grid, and as such, how smooth the auxiliary RHSs will effectively be. The latter, in turn, affect the quality of the discrete lacunae, i.e., how sharp the aft fronts of the waves really are in the discrete framework. This is important because every time a retarded component is subtracted, see (27), we assume that what is being subtracted inside the lacuna, i.e., on the domain $S(t)$, is zero, or more precisely, a small quantity that converges to zero with the refinement of the grid; the convergence, however, hinges on the smoothness of the source terms. Besides having a potential effect on the error behavior, 
the width of the transition region also determines on how many grid nodes the auxiliary RHSs are supported. We remind that those RHSs basically control the extent of temporal nonlocality of the lacunae-based ABCs. The algorithm requires keeping them on the interval of length $T_{\text {int }}$, and as such, the more narrow the transition region is, the less additional storage is needed.

In Figures 3, 4, 5, and 6 we are showing similar pressure error profiles for the width of the transition region $\varepsilon=8,6,4$, and 2 grid cell sizes, respectively.

We observe that with the decrease of $\varepsilon$ the error behavior deteriorates, which is natural to expect. We also notice, though, that the deterioration is more visible on the coarser grids, whereas on the finest $256 \times 512$ grid it is much slower. The $256 \times 512$ error profile is still practically flat for $\varepsilon=8$, see Figure 3 , and it loses only one binary order of magnitude for $\varepsilon=6$ over the entire long interval of integration, see Figure 4. Even for a rather narrow transition region, $\varepsilon=4$, see Figure 5, the finest grid error only grows by less than a factor of 2 over the first half of the integration interval, which is still fifty times the time needed for the waves to cross the domain. The rates of error increase become practically equal on all grids only for the narrowest transition region that we have tried: $\varepsilon=2$, see Figure 6 . Having only two grid cells in the transition region basically implies that there is no smoothing at all, rather a sharp truncation, and instead of $\mu(\boldsymbol{x}, t)$ we are using an equivalent of the Heaviside function on the grid. But even in this case we can see that the initial jump of the error is much smaller on the fine grid than on the coarser grids.

As of yet, we cannot offer a rigorous theoretical explanation of why the algorithm appears more sensitive to the quality of the discrete lacunae on coarser grids than on the fine grid. We can only qualitatively suggest that it has to do with the actual magnitude of those discrete "tails" behind the aft fronts of the waves that are due to the "imperfections" in the auxiliary sources, and that apparently are still smaller on fine grids. Altogether, this phenomenon is certainly beneficial, because fine grids are needed for high overall accuracy anyway, and at the same time they will allow to maintain high accuracy of the boundary treatment for longer periods of time. We should also note that for many practical applications the actual integration times will likely be not as long as those that we have used for the current proof-of-concept. This will leave even less room for the error buildup due to the boundary treatment.

\section{Conclusions}

We have constructed and tested the algorithm for setting highly-accurate global artificial boundary conditions for the computation of time-dependent acoustic waves. This work is an extension of our previous approach that applied 


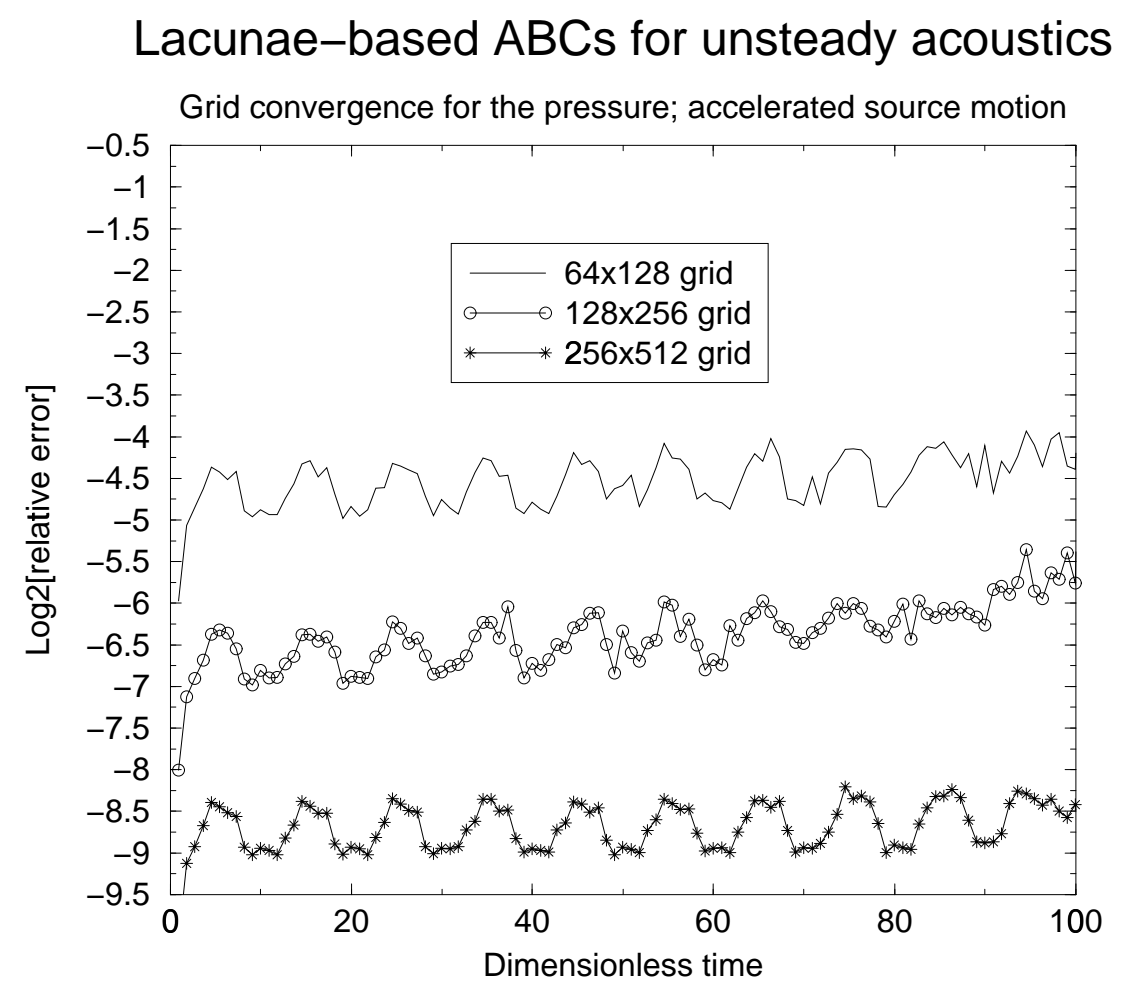

Fig. 3. Grid convergence study with lacunae-based ABCs, $\varepsilon=8 \Delta r$.

\section{Lacunae-based ABCs for unsteady acoustics}

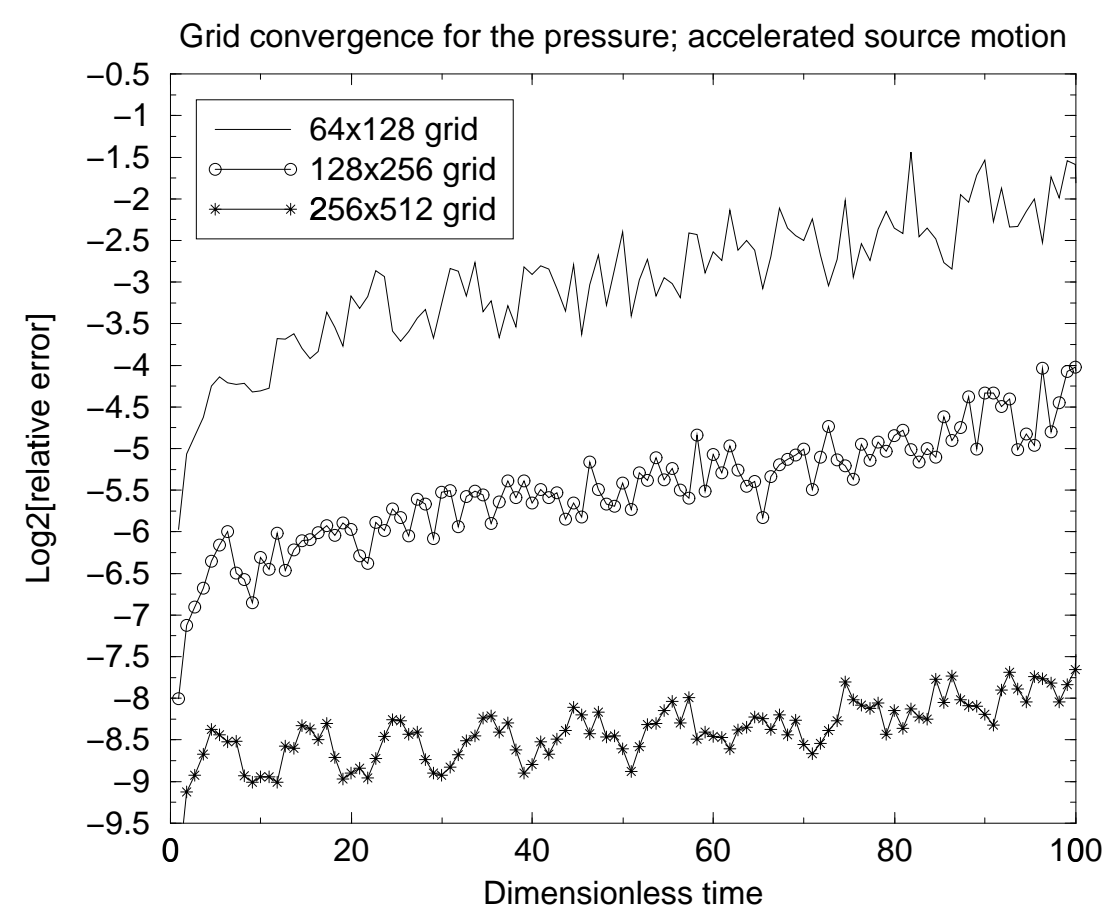

Fig. 4. Grid convergence study with lacunae-based ABCs, $\varepsilon=6 \Delta r$. 


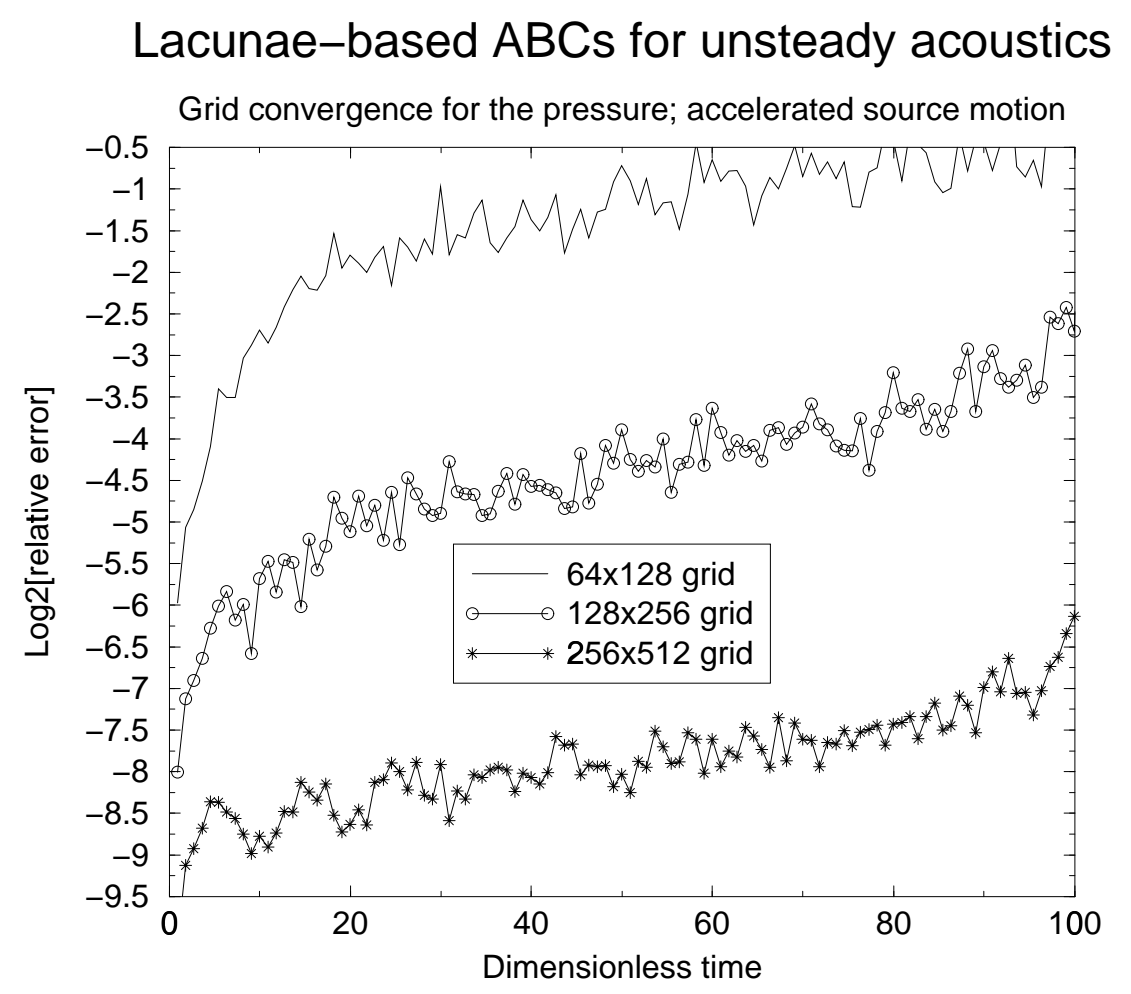

Fig. 5. Grid convergence study with lacunae-based ABCs, $\varepsilon=4 \Delta r$.

\section{Lacunae-based ABCs for unsteady acoustics}

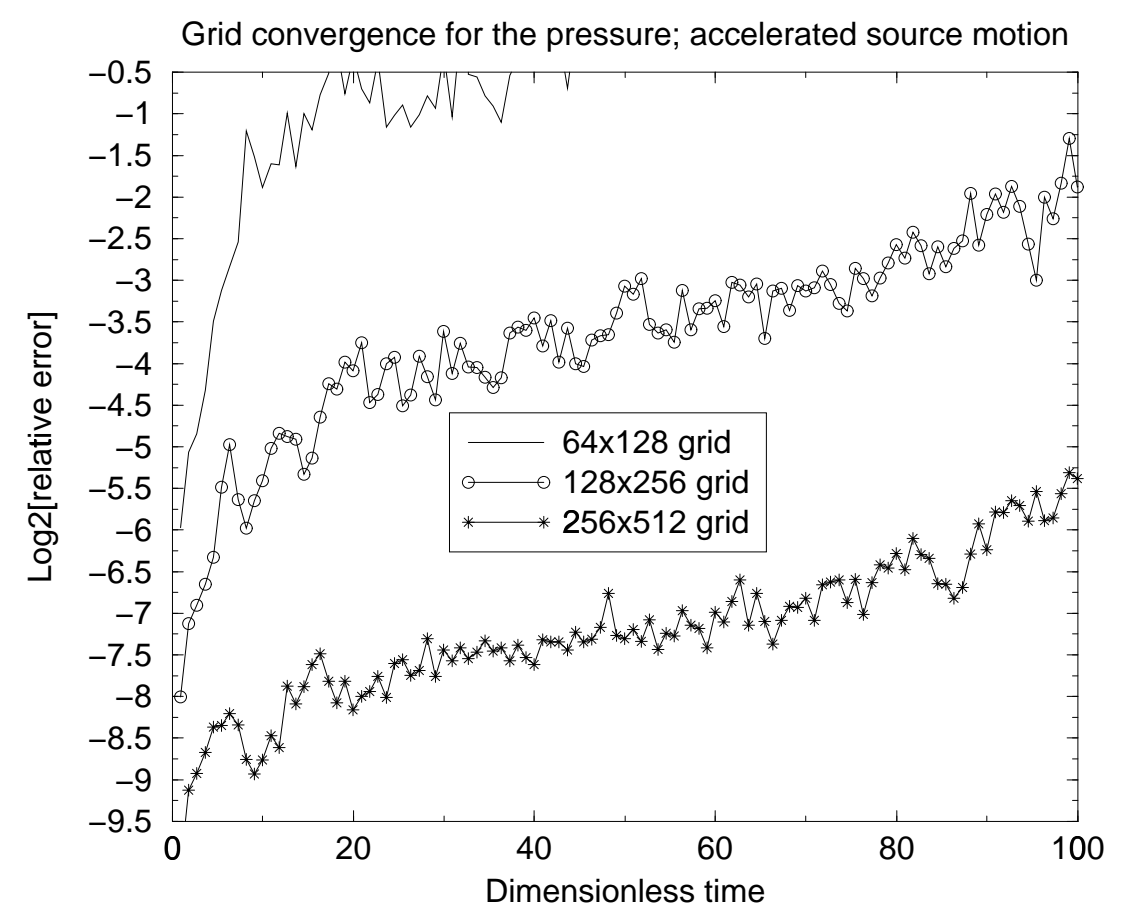

Fig. 6. Grid convergence study with lacunae-based ABCs, $\varepsilon=2 \Delta r$. 
to the scalar wave equation [13]. The algorithm is based on the presence of lacunae (aft fronts of the waves) in the three-dimensional wave-type solutions. The ABCs are obtained directly for the discrete formulation of the problem and can complement any consistent and stable finite-difference scheme. In so doing, neither a rational approximation of non-reflecting kernels, nor discretization of the continuous boundary conditions is required. The extent of temporal nonlocality of the new ABCs appears fixed and limited, and this is not a result of any approximation but rather a direct consequence of the fundamental properties of the solution. The proposed ABCs can handle artificial boundaries of irregular shape on regular grids with no fitting/adaptation needed. Besides, they possess a unique capability of being able to handle boundaries of moving computational domains, including the case of accelerated motion.

For the case of an acoustic source engaged in an accelerated motion, we have conducted numerical experiments that corroborate the theoretical design properties of the algorithm. We are currently unaware of any other acoustic ABCs' algorithm in the literature with the capability of handling the accelerated motion. We have also shown experimentally that when a key parameter that characterizes the algorithm changes so that to reduce the overall memory requirements, the performance of the ABCs suffers in an expected way. However, the deterioration of the long-term performance on fine grids is much slower than that on coarse grids.

Finally, we should mention that even though the full description of the algorithm provided in the paper does address a number of technical issues, its key idea is most straightforward. In one sentence it can be formulated as follows: One should not continue the computation inside the lacuna once the solution has become zero there due to "natural causes." Otherwise the error may unwarrantably build up on one hand, and on the other hand, the extent of the required temporal pre-history of the solution may grow un-justifiably high. The technical issues that we have discussed relate primarily to what to do with the waves outside, and not inside, the domain of interest. In the framework of the previous analysis, we simply allow them to propagate a certain distance away till they get reflected, and then set up the auxiliary domain so that the reflected waves do not reach the domain of interest before it completely falls inside the lacuna. This, however, is by no means the only possible option. In fact, any treatment of the outgoing waves that would prevent the reflections from re-entering the domain of interest before it falls into the lacuna will be appropriate. At the same time, introducing an alternative to the approach described in the current paper may be beneficial from the standpoint of the overall computational cost. For example, a treatment of the sponge layer type that slows down the outgoing waves, see, e.g., [25,26], may allow to reduce the size of the auxiliary domain. Alternatively, the lacunae-based approach can be combined with a PML-based treatment for the waves outside the domain of interest, see, e.g., the survey papers $[27,28]$. In any event, linearity has 
to be maintained, otherwise it will not be possible to partition the problem similarly to (16), (18), (19). These combined approaches will be subject of a future study.

\section{Acknowledgements}

The author is very thankful to the reviewers of the paper for their helpful comments.

\section{References}

[1] D. Givoli, Non-reflecting boundary conditions, J. Comput. Phys. 94 (1991) 129.

[2] T. Hagstrom, Radiation boundary conditions for the numerical simulation of waves, in: A. Iserlis (Ed.), Acta Numerica, Vol. 8, Cambridge University Press, Cambridge, 1999, pp. 47-106.

[3] S. V. Tsynkov, Numerical solution of problems on unbounded domains. A review, Appl. Numer. Math. 27 (1998) 465-532.

[4] M. J. Grote, J. B. Keller, On nonreflecting boundary conditions, J. Comput. Phys. 122 (1995) 231-243.

[5] M. J. Grote, J. B. Keller, Exact nonreflecting boundary conditions for the timedependent wave equation, SIAM J. Appl. Math. 55 (1995) 280-297.

[6] M. J. Grote, J. B. Keller, Nonreflecting boundary conditions for time-dependent scattering, J. Comput. Phys. 127 (1996) 52-65.

[7] I. L. Sofronov, Artificial boundary conditions of absolute transparency for twoand three-dimensional external time-dependent scattering problems, European J. Appl. Math. 9 (1998) 561-588.

[8] I. L. Sofronov, Non-reflecting inflow and outflow in a wind tunnel for transonic time-accurate simulations, J. Math. Anal. Appl. 221 (1998) 92-115.

[9] B. Alpert, L. Greengard, T. Hagstrom, Rapid evaluation of nonreflecting boundary kernels for time-domain wave propagation, SIAM J. Numer. Anal. 37 (2000) 1138-1164.

[10] B. Alpert, L. Greengard, T. Hagstrom, Nonreflecting boundary conditions for the time-dependent wave equation, J. Comput. Phys. 180 (1) (2002) 270-296.

[11] E. Michielssen, A. Ergin, B. Shanker, D. Weile, The multilevel plane wave time domain algorithm and its applications to the rapid solution of electromagnetic 
scattering problems: A review, in: Mathematical and numerical aspects of wave propagation (Santiago de Compostela, 2000), SIAM, Philadelphia, PA, 2000, pp. $24-33$.

[12] V. S. Ryaben'kii, S. V. Tsynkov, V. I. Turchaninov, Long-time numerical computation of wave-type solutions driven by moving sources, Appl. Numer. Math. 38 (2001) 187-222.

[13] V. S. Ryaben'kii, S. V. Tsynkov, V. I. Turchaninov, Global discrete artificial boundary conditions for time-dependent wave propagation, J. Comput. Phys. 174 (2) (2001) 712-758.

[14] V. S. Ryaben'kii, Nonreflecting time-dependent boundary conditions on artificial boundaries of varying location and shape, Appl. Numer. Math. 33 (2000) 481-492.

[15] V. S. Vladimirov, Equations of Mathematical Physics, Dekker, New-York, 1971.

[16] I. Petrowsky, On the diffusion of waves and the lacunas for hyperbolic equations, Matematicheskii Sbornik (Recueil Mathématique) 17 (59) (3) (1945) 289-370.

[17] R. Courant, D. Hilbert, Methods of Mathematical Physics. Volume II, Wiley, New York, 1962.

[18] L. D. Landau, E. M. Lifshitz, Fluid Mechanics, Pergamon Press, Oxford, 1986.

[19] C. L. Morfey, Dictionary of Acoustics, Academic Press, San Diego, 2001.

[20] L. Ting, M. J. Miksis, Exact boundary conditions for scattering problems, J. Acoust. Soc. Am. 80 (1986) 1825-1827.

[21] D. Givoli, D. Cohen, Nonreflecting boundary conditions based on Kirchhoff-type formulae, J. Comput. Phys. 117 (1995) 102-113.

[22] S. V. Tsynkov, External boundary conditions for three-dimensional problems of computational aerodynamics, SIAM J. Sci. Comp. 21 (1999) 166-206.

[23] P. M. Morse, H. Feshbach, Methods of Theoretical Physics. Parts I and II, McGraw-Hill, Boston, 1953.

[24] K. S. Yee, Numerical solution of initial boundary value problem involving Maxwell's equations in isotropic media, IEEE Trans. Antennas Propagat. 14 (1966) 302-307.

[25] M. Israeli, S. Orszag, Approximation of radiation boundary conditions, J. Comput. Phys 41 (1981) 115-135.

[26] S. Karni, Far-field filtering operators for suppression of reflections from artificial boundaries, SIAM J. Numer. Anal. 33 (1996) 1014-1047.

[27] S. Abarbanel, D. Gottlieb, On the construction and analysis of absorbing layers in CEM, Appl. Numer. Math. 27 (1998) 331-340.

[28] E. Turkel, A. Yefet, Absorbing PML boundary layers for wave-like equations, Appl. Numer. Math. 27 (1998) 533-557. 